\title{
The gluon and ghost propagators in Euclidean Yang-Mills theory in the maximal Abelian gauge: taking into account the effects of the Gribov copies and of the dimension two condensates
}

\author{
M.A.L. Capri ${ }^{a *}$, V.E.R. Lemes ${ }^{a \dagger}$, \\ R.F. Sobreiro ${ }^{b \ddagger}$, S.P. Sorella ${ }^{a \S}$, R. Thibes ${ }^{a \|}$ \\ ${ }^{a}$ UERJ - Universidade do Estado do Rio de Janeiro \\ Instituto de Física - Departamento de Física Teórica \\ Rua São Francisco Xavier 524, 20550-013 Maracanã, Rio de Janeiro, Brasil \\ ${ }^{b}$ CBPF - Centro Brasileiro de Pesquisas Físicas \\ Rua Xavier Sigaud 150, 22290-180, Urca, Rio de Janeiro, Brasil
}

\begin{abstract}
The infrared behavior of the gluon and ghost propagators is studied in $S U(2)$ Euclidean Yang-Mills theory in the maximal Abelian gauge within the Gribov-Zwanziger framework. The nonperturbative effects associated with the Gribov copies and with the dimension two condensates are simultaneously encoded into a local and renormalizable Lagrangian. The resulting behavior turns out to be in good agreement with the lattice data.
\end{abstract}

\section{Introduction}

The study of the infrared behavior of the gluon and ghost propagators has been the object of intensive investigations in recent years. Albeit not gauge invariant, these correlation functions enable us to probe the reliability of the various approaches which give rise to our current understanding of the behavior of Yang-Mills theories in the infrared, a task which is far from being achieved. This is due to the fact that propagators are the simplest Green's functions allowing us to evaluate in analytic form certain nonperturbative effects expected to be relevant in the infrared. Moreover, the lattice community has been able to develop accurate algorithms for a nonperturbative numerical study of the gluon and ghost propagators, which can be now analyzed on huge lattices, allowing therefore for a comparison between analytical and numerical results. Evidently, a qualitative agreement might be very encouraging in pursuing further investigations of our theoretical frameworks. We also underline that this possibility is not restricted to a particular gauge. Nowadays, the gluon and ghost propagators can be studied from both theoretical and numerical viewpoints in several gauges as, for example, the Landau, Coulomb and maximal Abelian gauge.

In this paper we focus on the gluon and ghost propagators in the maximal Abelian gauge [1, 2, 3], which we are investigating since several years $[4,5,6,7,8,9,10,11,12,13,14]$. In our previous works we have provided

\footnotetext{
*marcio@dft.if.uerj.br

$\dagger$ †itor@dft.if.uerj.br

${ }^{\ddagger}$ sobreiro@cbpf.br

$\S$ sorella@uerj.br

ף Work supported by FAPERJ, Fundação de Amparo à Pesquisa do Estado do Rio de Janeiro, under the program Cientista do Nosso Estado, E-26/100.615/2007.

" thibes@dft.if.uerj.br
} 
analytical evidence of nonperturbative effects which should be taken into account when facing the various features displayed by this gauge, such as the dual superconductivity picture for color confinement [15] and the Abelian dominance hypothesis $[16,17,18,19]$. It turns out that these nonperturbative effects can be accounted for by a set of dimension two operators which can be consistently introduced in the Yang-Mills action.

As other Lorentz covariant gauges, the maximal Abelian gauge is plagued by the existence of Gribov copies [20], requiring that the domain of integration in the Feynman path integral has to be suitably restricted to the so called Gribov region [21]. As discussed in [10,13], this restriction can be implemented by introducing a dimension two nonlocal operator, known as the horizon function, namely

$$
S_{\mathrm{hor}}=\gamma^{4} g^{2} \int d^{4} x \varepsilon^{a b} A_{\mu}\left(\mathcal{M}^{-1}\right)^{a c} \varepsilon^{c b} A_{\mu},
$$

where $\gamma$ is the Gribov parameter ${ }^{1}$ and $\left(\mathcal{M}^{-1}\right)^{a b}$ is the inverse of Faddeev-Popov operator

$$
\mathcal{M}^{a b}=-D_{\mu}^{a c} D_{\mu}^{c b}-g^{2} \varepsilon^{a c} \varepsilon^{b d} A_{\mu}^{c} A_{\mu}^{d},
$$

with $A_{\mu}$ and $A_{\mu}^{a}$ being the diagonal and off-diagonal components of the gauge field, respectively, i.e. $A_{\mu}=A_{\mu}^{3}$ and $a=1,2$. Expression (1) generalizes to the maximal Abelian gauge the horizon function already obtained by Zwanziger $[22,23]$ in the Landau gauge. The nonlocal operator (1) can be localized by means of the introduction of a set of auxiliary fields $\left(\bar{\phi}_{i}^{a}, \phi_{i}^{a}, \bar{\omega}_{i}^{a}, \omega_{i}^{a}\right)$, so that the resulting action enjoys renormalizability [10, 13].

In addition of the horizon function, other dimension two operators have been investigated. Our results have given support to the fact that the preferred vacuum state is that in which those operators condense, i.e. they develop a nonvanishing vacuum expectation value, lowering the vacuum energy of the theory. The first dimension two operator which has been studied is the gluon operator $A_{\mu}^{a} A_{\mu}^{a}$. This operator turns out to be multiplicatively renormalizable $[24,7]$ and its condensation, i.e. $\left\langle A_{\mu}^{a} A_{\mu}^{a}\right\rangle \neq 0$, gives rise to a dynamical mass generation for offdiagonal gluons [8], in agreement with the Abelian dominance hypothesis. As second example of dimension two operator let us quote the ghost operator $\varepsilon^{a b} \bar{c}^{a} c^{b}$, where $\bar{c}^{a}, c^{a}$ denote the off-diagonal Faddeev-Popov ghosts. This operator is responsible for the spontaneous breaking of the global $S L(2, \mathbb{R})$ symmetry present in the ghost sector of the maximal Abelian gauge. It has been investigated by several authors [25, 26, 5, 27], see for instance ref.[14] for a recent analysis of its renormalizability as well as of its condensation. The third dimension two operator which we shall consider is given by $\left(\bar{\phi}_{i}^{a} \phi_{i}^{a}-\bar{\omega}_{i}^{a} \omega_{i}^{a}-\bar{c}^{a} c^{a}\right)$. It generalizes to the maximal Abelian gauge the operator introduced recently in the case of the Landau gauge [28]. It reflects the nontrivial dynamics developed by the interacting auxiliary fields $\left(\bar{\phi}_{i}^{a}, \phi_{i}^{a}, \bar{\omega}_{i}^{a}, \omega_{i}^{a}\right)$ needed to localize the horizon term (1).

However, so far, these dimension two operators have not yet been analyzed simultaneously, a necessary step in order to get a more precise idea of their relevance in the infrared. This was due to the nontrivial task of explicitly constructing them, as in the case of the horizon function, eq.(1), as well as to the need of establishing their renormalizability properties. The aim of this paper is that of filling this gap, by presenting a detailed analysis of the gluon and ghost propagators when all these dimension two operators are present in the starting action. In a sense, the present work can be seen as a kind of summary of our efforts towards a better understanding of the infrared behavior of the gluon and ghost propagators in the maximal Abelian gauge.

The output of our results can be summarized as follows:

- when all dimension two operators are simultaneously taken into account, the resulting local action remains renormalizable. This nontrivial feature is due to the large set of Ward identities which can be established when all operators are present.

- the resulting behavior of the gluon and ghost propagators turns out to be in remarkable agreement with the available lattice data $[29,30,31]$. It is worth underlining that all dimension two operators affect the propagators. In other words, such a behavior of the gluon and ghost propagators can be obtained only when the dimension two operators are simultaneously taken into account.

\footnotetext{
${ }^{1}$ We remind that the Gribov parameter $\gamma$ is not a free parameter. It is determined by the gap equation $\frac{\delta \Gamma}{\delta \gamma}=0$, where $\Gamma$ is the $1 \mathrm{PI}$ quantum effective action evaluated by taking as starting point the Yang-Mills action with the addition of the horizon term [22, 23]. As such, the parameter $\gamma$ can be expressed in terms of the gauge coupling constant and of the invariant scale $\Lambda_{Q C D}$.
} 
The work is organized as follows. In order to provide a more easy reading of the paper, in Sect.2 we give a general overview of our results about the gluon and ghost propagators, providing a comparison with the recent lattice data. In Sect. 3 we present a detailed discussion of the inclusion in the starting action of the aforementioned dimension two operators. In Sect.4 we derive the set of Ward identities fulfilled by the complete action. In Sect.5 we address the issue of the renormalizability of the model. Sect. 6 collects our conclusion.

\section{Summary of the results}

\subsection{Notation}

Let us start by briefly reminding the standard notation in the case of the maximal Abelian gauge. The gauge field $\mathcal{A}_{\mu}$ is decomposed as

$$
\mathcal{A}_{\mu}=A_{\mu}^{A} T^{A} \equiv A_{\mu}^{a} T^{a}+A_{\mu} T^{3}
$$

The generator $T^{3}$ stands for the diagonal generator of the $U(1)$ Cartan subalgebra of $S U(2)$, while the index $a=1,2$ labels the remaining off-diagonal generators $\left\{T^{a}\right\}$.

Accordingly, the field strength decomposes as

$$
\begin{aligned}
F_{\mu \nu}^{a} & =D_{\mu}^{a b} A_{\nu}^{b}-D_{\nu}^{a b} A_{\mu}^{b}, \\
F_{\mu \nu}^{3} & \equiv F_{\mu \nu}=\partial_{\mu} A_{\nu}-\partial_{\nu} A_{\mu}+g \varepsilon^{a b} A_{\mu}^{a} A_{\nu}^{b} \\
\varepsilon^{a b} & \equiv \varepsilon^{3 a b},
\end{aligned}
$$

where we have introduced the covariant derivative $D_{\mu}^{a b}$ with respect to the diagonal components $A_{\mu}$ of the gauge field, namely

$$
D_{\mu}^{a b} \equiv \delta^{a b} \partial_{\mu}-g \varepsilon^{a b} A_{\mu}
$$

\subsection{The tree level gluon and ghost propagators}

We collect here our results for the gluon and ghost propagators.

\section{- The off-diagonal gluon propagator:}

the transverse off-diagonal gluon propagator turns out to be of the Yukawa type

$$
\left\langle A_{\mu}^{a}(-k) A_{\nu}^{b}(k)\right\rangle=\frac{1}{k^{2}+m^{2}}\left(\delta_{\mu \nu}-\frac{k_{\mu} k_{\nu}}{k^{2}}\right) \delta^{a b},
$$

where $m$ is the dynamical mass originating from the condensation of the gluon operator [8]

$$
\mathcal{O}_{A^{2}}=\frac{1}{2} A_{\mu}^{a} A_{\mu}^{a}
$$

This behavior has been reported in lattice simulations [29, 30, 31]. It supports the Abelian dominance hypothesis, according to which the off-diagonal gluons should acquire a sufficiently large dynamical mass which decouple them at low energies.

\section{- The diagonal gluon propagator:}

for the diagonal gluon propagator we have obtained an infrared suppressed propagator of the Gribov-Stingl type, namely

$$
\left\langle A_{\mu}(-k) A_{\nu}(k)\right\rangle=\frac{k^{2}+\mu^{2}}{k^{4}+\mu^{2} k^{2}+4 \gamma^{4} g^{2}}\left(\delta_{\mu \nu}-\frac{k_{\mu} k_{\nu}}{k^{2}}\right),
$$


where $\gamma$ is the Gribov parameter and $\mu$ is a mass parameter related to the condensation of the operator [32]

$$
\mathcal{O}_{\bar{f} f}=\left(\bar{\phi}_{i}^{a} \phi_{i}^{a}-\bar{\omega}_{i}^{a} \omega_{i}^{a}-\bar{c}^{a} c^{a}\right) .
$$

We observe that expression (8) does not vanish at the origin, in full agreement with the recent numerical data [31]. It gives rise to a positivity violating propagator in configuration space, a feature usually interpreted as evidence for gluon confinement.

Moreover, it is worth to point out that the diagonal gluon propagator, (8), can be naturally rewritten in terms of a power-law dynamical running mass of the type

$$
\left\langle A_{\mu}(-k) A_{\nu}(k)\right\rangle=\frac{1}{k^{2}+M^{2}(k)}\left(\delta_{\mu \nu}-\frac{k_{\mu} k_{\nu}}{k^{2}}\right),
$$

where

$$
M^{2}(k)=\frac{4 \gamma^{4} g^{2}}{k^{2}+\mu^{2}} .
$$

Expression (10) is in accordance with the definition firstly envisaged in references [33, 34] and subsequently found in the operator product expansion (OPE) approach by [35], and later in Schwinger-Dyson equations by $[36]$.

\section{- The symmetric off-diagonal ghost propagator:}

for the symmetric off-diagonal ghost propagator we have found

$$
\left\langle\bar{c}^{a}(-k) c^{b}(k)\right\rangle_{\mathrm{symm}}=\frac{k^{2}+\mu^{2}}{k^{4}+2 \mu^{2} k^{2}+\left(\mu^{4}+v^{4}\right)} \delta^{a b},
$$

where $v$ is a mass parameter related to the condensation of the ghost operator [14]

$$
\mathcal{O}_{\text {ghost }}=g \varepsilon^{a b} \bar{c}^{a} c^{b} .
$$

Notice that expression (12) is suppressed in the infrared and attains a nonvanishing finite value at $k=0$. Again, this behavior agrees with that reported in [31].

\section{- The antisymmetric off-diagonal ghost propagator:}

finally, for the antisymmetric off-diagonal ghost propagator we have

$$
\left\langle\bar{c}^{a}(-k) c^{b}(k)\right\rangle_{\text {antisymm }}=\frac{v^{2}}{k^{4}+2 \mu^{2} k^{2}+\left(\mu^{4}+v^{4}\right)} \varepsilon^{a b} .
$$

As expected, this behavior is a consequence of the ghost condensate [14], $\left\langle\varepsilon^{a b} \bar{c}^{a} c^{b}\right\rangle \sim v^{2}$, being in agreement with [31].

In summary, the behavior shown above for the gluon and ghost propagators turns out to be in remarkable agreement with the most recent lattice data, as reported in [31]. This can be taken as a useful indication of the fact that the aforementioned dimension two operators play a relevant role in the infrared. Let also underline that all mass parameters, $(m, \gamma, \mu, v)$, entering the gluon and ghost propagators are not free parameters, being determined in a dynamical way as solutions of gap equations, obtained by minimizing the vacuum energy, see for instance refs. $[14,8]$ for an estimate of the values of $m$ and $v$ at one-loop order. As such, all parameters will get proportional to the unique scale of the theory, i.e. $(m, \gamma, \mu, v) \propto \Lambda_{Q C D}$. 


\section{Identification of the complete classical action}

\subsection{The Yang-Mills action and the gauge fixing term}

In order to obtain the complete classical action, let us start by specifying the gauge fixing term, namely

$$
S_{0}=S_{\mathrm{YM}}+S_{\mathrm{MAG}}
$$

where $S_{\mathrm{YM}}$ is the Yang-Mills action in Euclidean spacetime

$$
S_{\mathrm{YM}}=\frac{1}{4} \int d^{4} x\left(F_{\mu \nu}^{a} F_{\mu \nu}^{a}+F_{\mu \nu} F_{\mu \nu}\right)
$$

with $F_{\mu \nu}^{a}, F_{\mu \nu}$ and $D_{\mu}^{a b}$ given in eqs.(4),(5). The term $S_{\mathrm{MAG}}$ in expression (15) stands for the gauge fixing term of the maximal Abelian gauge, being given by

$$
S_{\mathrm{MAG}}=\int d^{4} x\left[i b^{a} D_{\mu}^{a b} A_{\mu}^{b}-\bar{c}^{a} \mathcal{M}^{a b} c^{b}+g \varepsilon^{a b} \bar{c}^{a}\left(D_{\mu}^{b c} A_{\mu}^{c}\right) c+i b \partial_{\mu} A_{\mu}+\bar{c} \partial_{\mu}\left(\partial_{\mu} c+g \varepsilon^{a b} A_{\mu}^{a} c^{b}\right)\right],
$$

where $\left(b^{a}, b\right)$ are the off-diagonal and diagonal Lagrange multipliers enforcing the gauge conditions, given by $D_{\mu}^{a b} A_{\mu}^{b}=0$ and $\partial_{\mu} A_{\mu}=0$. The fields $\left(c^{a}, \bar{c}^{a}, c, \bar{c}\right)$ are the off-diagonal and diagonal Faddeev-Popov ghosts, respectively, and $\mathcal{M}^{a b}$ denotes the Faddeev-Popov operator

$$
\mathcal{M}^{a b}=-D_{\mu}^{a c} D_{\mu}^{c b}-g^{2} \varepsilon^{a c} \varepsilon^{b d} A_{\mu}^{c} A_{\mu}^{d}
$$

The action (15) is left invariant by the nilpotent BRST transformation

$$
\begin{aligned}
s^{2}=0, & \\
s A_{\mu}^{a}=-\left(D_{\mu}^{a b} c^{b}+g \varepsilon^{a b} A_{\mu}^{b} c\right), & s A_{\mu}=-\left(\partial_{\mu} c+g \varepsilon^{a b} A_{\mu}^{a} c^{b}\right), \\
s c^{a}=g \varepsilon^{a b} c^{b} c, & s c=\frac{g}{2} \varepsilon^{a b} c^{a} c^{b}, \\
s \bar{c}^{a}=i b^{a}, & s \bar{c}=i b, \\
s b^{a}=0, & s b=0 .
\end{aligned}
$$

Notice that the gauge fixing term (17) can be written as a pure BRST variation

$$
S_{\mathrm{MAG}}=s \int d^{4} x\left(\bar{c}^{a} D_{\mu}^{a b} A_{\mu}^{b}+\bar{c} \partial_{\mu} A_{\mu}\right)
$$

\subsection{Introduction of the horizon function, localization, and softly broken BRST in- variance}

As already mentioned, the maximal Abelian gauge is affected by the existence of Gribov copies, which have to be taken into account in order to properly quantize the theory. To deal with this problem it is necessary to restrict the domain of integration in the Feynman path integrals to the so-called Gribov region $\Omega$. In the case of the maximal Abelian gauge, this region is defined as [10,13]

$$
\Omega=\left\{\left(A_{\mu}^{a}, A_{\mu}\right), \quad D_{\mu}^{a b} A_{\mu}^{b}=0, \partial_{\mu} A_{\mu}=0, \mathcal{M}^{a b}=-D_{\mu}^{a c} D_{\mu}^{c b}-g^{2} \varepsilon^{a c} \varepsilon^{b d} A_{\mu}^{c} A_{\mu}^{d}>0\right\} .
$$

The restriction of the domain of integration is achieved through the introduction of the horizon function $S_{\text {hor }}$, eq.(1). Therefore, for the partition function we write $[10,13]$

$$
\mathcal{Z}=\int[d A][d b][d \bar{c}][d c] e^{-\left(S_{\mathrm{YM}}+S_{\mathrm{MAG}}+S_{\mathrm{hor}}\right)}
$$


The nonlocal term $S_{\text {hor }}$ can be localized by means of a pair of complex vector bosonic fields, $\left(\phi_{\mu}^{a b}, \bar{\phi}_{\mu}^{a b}\right)$ according to

$$
e^{-S_{\text {hor }}}=\int[d \bar{\phi}][d \phi](\operatorname{det} \mathcal{M})^{8} \exp \left\{-\int d^{4} x\left[\bar{\phi}_{\mu}^{a b} \mathcal{M}^{a c} \phi_{\mu}^{a b}+\gamma^{2} g \varepsilon^{a b}\left(\phi_{\mu}^{a b}-\bar{\phi}_{\mu}^{a b}\right) A_{\mu}\right]\right\},
$$

where the determinant, $(\operatorname{det} \mathcal{M})^{8}$, takes into account the Jacobian arising from the integration over the fields $\left(\phi_{\mu}^{a b}, \bar{\phi}_{\mu}^{a b}\right)$. This term can also be localized by means of a pair of complex vector anticommuting fields $\left(\omega_{\mu}^{a b}, \bar{\omega}_{\mu}^{a b}\right)$, namely

$$
(\operatorname{det} \mathcal{M})^{8}=\int[d \bar{\omega}][d \omega] \exp \left(\int d^{4} x \bar{\omega}_{\mu}^{a b} \mathcal{M}^{a c} \omega_{\mu}^{c b}\right)
$$

Therefore, the horizon function gives place to a local term $S_{\text {Local }}$, namely

$$
\begin{aligned}
e^{-S_{\text {hor }}} & =\int[d \bar{\phi}][d \phi][d \bar{\omega}][d \omega] e^{-S_{\text {Local }}}, \\
S_{\text {Local }} & =\int d^{4} x\left[\bar{\phi}_{\mu}^{a b} \mathcal{M}^{a c} \phi_{\mu}^{c b}-\bar{\omega}_{\mu}^{a b} \mathcal{M}^{a c} \omega_{\mu}^{c b}+\gamma^{2} g \varepsilon^{a b}\left(\phi_{\mu}^{a b}-\bar{\phi}_{\mu}^{a b}\right) A_{\mu}\right] .
\end{aligned}
$$

Following [22, 23], we introduce the BRST transformations of the localizing fields $\left(\phi_{\mu}^{a b}, \bar{\phi}_{\mu}^{a b}\right)$ and $\left(\omega_{\mu}^{a b}, \bar{\omega}_{\mu}^{a b}\right)$ as

$$
\begin{array}{ll}
s \phi_{\mu}^{a b}=\omega_{\mu}^{a b}, & s \omega_{\mu}^{a b}=0, \\
s \bar{\omega}_{\mu}^{a b}=\bar{\phi}_{\mu}^{a b}, & s \bar{\phi}_{\mu}^{a b}=0 .
\end{array}
$$

It should be noted, however, that expression $S_{\text {Local }}$ does not exhibit BRST invariance, which turns out to be broken by soft terms proportional to the Gribov parameter $\gamma$. In fact

$$
s \int d^{4} x \gamma^{2} g \varepsilon^{a b}\left(\phi_{\mu}^{a b}-\bar{\phi}_{\mu}^{a b}\right) A_{\mu}=\gamma^{2} g \int d^{4} x\left[\varepsilon^{a b} \omega_{\mu}^{a b} A_{\mu}-\varepsilon^{a b}\left(\phi_{\mu}^{a b}-\bar{\phi}_{\mu}^{a b}\right)\left(\partial_{\mu} c+g \varepsilon^{m n} A_{\mu}^{m} c^{n}\right)\right] \text {. }
$$

Nevertheless, as in the case of the Landau gauge $[22,23,37]$, the soft breaking (28) does not spoil the renormalizability of the theory $[10,13]$. This remarkable feature relies on the possibility of extending to the maximal Abelian gauge the same procedure outlined by Zwanziger in the case of the Landau gauge [22, 23], amounting to embed $S_{\text {Local }}$ into a more general action, $S_{\text {Local }}^{\text {inv }}$, which enjoys exact BRST invariance, namely

$$
S_{\text {Local }} \rightarrow S_{\text {Local }}^{\text {inv }}, \quad s S_{\text {Local }}^{\text {inv }}=0
$$

Furthermore, as it will be shown below, the term $S_{\text {Local }}$ can be easily recovered from $S_{\text {Local }}^{\text {inv }}$ The manifest BRST invariant action $S_{\text {Local }}^{\text {inv }}$ is found to be $[10,13]$

$$
\begin{aligned}
S_{\text {Local }}^{\text {inv }}= & s \int d^{4} x\left(\bar{\omega}_{\mu}^{a b} \mathcal{M}^{a c} \phi_{\mu}^{c b}-\bar{N}_{\mu \nu}^{a b} D_{\mu}^{a c} \phi_{\nu}^{c b}+M_{\mu \nu}^{a b} D_{\mu}^{a c} \bar{\omega}_{\nu}^{c b}\right) \\
= & \int d^{4} x\left\{\bar{\phi}_{\mu}^{a b} \mathcal{M}^{a c} \phi_{\mu}^{c b}-\bar{\omega}_{\mu}^{a b} \mathcal{M}^{a c} \omega_{\mu}^{c b}+\bar{\omega}_{\mu}^{a b} \mathcal{F}^{a c} \phi_{\mu}^{c b}+\bar{M}_{\mu \nu}^{a b} D_{\mu}^{a c} \phi_{\nu}^{c b}+N_{\mu \nu}^{a b} D_{\mu}^{a c} \bar{\omega}_{\nu}^{c b}\right. \\
& \left.+\bar{N}_{\mu \nu}^{a b}\left[D_{\mu}^{a c} \omega_{\nu}^{c b}+g \varepsilon^{a c}\left(\partial_{\mu} c+g \varepsilon^{d e} A_{\mu}^{d} c^{e}\right) \phi_{\nu}^{c b}\right]+M_{\mu \nu}^{a b}\left[D_{\mu}^{a c} \bar{\phi}_{\nu}^{c b}+g \varepsilon^{a c}\left(\partial_{\mu} c+g \varepsilon^{d e} A_{\mu}^{d} c^{e}\right) \bar{\omega}_{\nu}^{c b}\right]\right\}
\end{aligned}
$$

where

$$
\mathcal{F}^{a b}=2 g \varepsilon^{a c}\left(\partial_{\mu} c+g \varepsilon^{d e} A_{\mu}^{d} c^{e}\right) D_{\mu}^{c b}+g \varepsilon^{a b} \partial_{\mu}\left(\partial_{\mu} c+g \varepsilon^{c d} A_{\mu}^{c} c^{d}\right)-g^{2}\left(\varepsilon^{a c} \varepsilon^{b d}+\varepsilon^{a d} \varepsilon^{b c}\right) A_{\mu}^{d}\left(D_{\mu}^{c e} c^{e}+g \varepsilon^{c e} A_{\mu}^{e} c\right)
$$

and the external sources $\left(M_{\mu \nu}^{a b}, \bar{M}_{\mu \nu}^{a b}\right),\left(N_{\mu \nu}^{a b}, \bar{N}_{\mu \nu}^{a b}\right)$ transform as

$$
\begin{array}{ll}
s M_{\mu \nu}^{a b}=N_{\mu \nu}^{a b}, & s N_{\mu \nu}^{a b}=0, \\
s \bar{N}_{\mu \nu}^{a b}=-\bar{M}_{\mu \nu}^{a b}, & s \bar{M}_{\mu \nu}^{a b}=0 .
\end{array}
$$

In order to reobtain $S_{\text {Local }}$ by the BRST invariant action $S_{\text {Local }}^{\text {inv }}$ we first take the physical limits of the external sources $\left(M_{\mu \nu}^{a b}, \bar{M}_{\mu \nu}^{a b}\right),\left(N_{\mu \nu}^{a b}, \bar{N}_{\mu \nu}^{a b}\right)$, which are defined by $[10,13]$

$$
\begin{aligned}
& \left.M_{\mu \nu}^{a b}\right|_{\text {phys }}=-\left.\bar{M}_{\mu \nu}^{a b}\right|_{\text {phys }}=-\delta^{a b} \delta_{\mu \nu} \gamma^{2}, \\
& \left.N_{\mu \nu}^{a b}\right|_{\text {phys }}=-\left.\bar{N}_{\mu \nu}^{a b}\right|_{\text {phys }}=0,
\end{aligned}
$$


and then perform a shift in the variable $\omega_{\mu}^{a b}$ as $[10,13]$

$$
\omega_{\mu}^{a b} \rightarrow \omega_{\mu}^{a b}+\left(\mathcal{M}^{-1}\right)^{a c}\left[\mathcal{F}^{c d} \phi_{\mu}^{d b}+\gamma^{2} g \varepsilon^{c b}\left(\partial_{\mu} c+g \varepsilon^{d e} A_{\mu}^{d} c^{e}\right)\right],
$$

so that

$$
\left.S_{\text {Local }}^{\text {inv }}\right|_{\text {phys }}=S_{\text {Local }}
$$

Thus, we consider the following action

$$
S_{1}=S_{0}+S_{\text {Local }}^{\text {inv }},
$$

which enjoys the property of being BRST invariant,

$$
s S_{1}=0 .
$$

\subsection{Inclusion of the quartic ghost term}

Albeit BRST invariant, the action $S_{1}$ is not yet the most general classical action to start with. The nonlinearity of the gauge condition, $D_{\mu}^{a b} A_{\mu}^{b}=0$, requires the introduction of a quartic term in the Faddeev-Popov ghost fields

$$
\frac{g^{2}}{2} \bar{c}^{a} c^{a} \bar{c}^{b} c^{b}
$$

which is in fact needed for renormalization purposes. In our case, due to the presence of the localizing fields $\left(\phi_{\mu}^{a b}, \bar{\phi}_{\mu}^{a b}, \omega_{\mu}^{a b}, \bar{\omega}_{\mu}^{a b}\right)$, the quartic ghost term is introduced in a BRST invariant way through the following invariant action $S_{\alpha}$

$$
\begin{aligned}
S_{\alpha}= & -\frac{\alpha}{2} s \int d^{4} x\left[\bar{c}^{a} i b^{a}-g \varepsilon^{a b} \bar{c}^{a} \bar{c}^{b} c+g^{2} \bar{\omega}_{\mu}^{a b} \phi_{\mu}^{a b}\left(\bar{\phi}_{\nu}^{c d} \phi_{\nu}^{c d}-\bar{\omega}_{\nu}^{c d} \omega_{\nu}^{c d}\right)-2 g^{2} \bar{\omega}_{\mu}^{a c} \phi_{\mu}^{a c} \bar{c}^{b} c^{b}\right] \\
= & \frac{\alpha}{2} \int d^{4} x\left[b^{a} b^{a}+2 i g \varepsilon^{a b} b^{a} \bar{c}^{b} c-g^{2}\left(\bar{\phi}_{\mu}^{a b} \phi_{\mu}^{a b}-\bar{\omega}_{\mu}^{a b} \omega_{\mu}^{a b}-\bar{c}^{a} c^{a}\right)\left(\bar{\phi}_{\nu}^{c d} \phi_{\nu}^{c d}-\bar{\omega}_{\nu}^{c d} \omega_{\nu}^{c d}-\bar{c}^{c} c^{c}\right)\right. \\
& \left.-2 i g^{2} \bar{\omega}_{\mu}^{a c} \phi_{\mu}^{a c} b^{b} c^{b}+2 g^{3} \bar{\omega}_{\mu}^{a c} \phi_{\mu}^{a c} \varepsilon^{b d} \bar{c}^{b} c^{d} c\right],
\end{aligned}
$$

where $\alpha$ is a gauge parameter, which has to be set to zero after the renormalization procedure. In fact, introducing the action $S_{2}$ as

$$
S_{2}=S_{1}+S_{\alpha},
$$

it follows that the equation of motion of the off-diagonal Lagrange multiplier $b^{a}$ gets modified according to

$$
\frac{\delta S_{2}}{\delta b^{a}}=i D_{\mu}^{a b} A_{\mu}^{b}+\alpha\left(b^{a}+i g \varepsilon^{a b} \bar{c}^{b} c-i g^{2} \bar{\omega}_{\mu}^{b c} \phi_{\mu}^{b c} c^{a}\right) .
$$

Therefore, one can see that the gauge condition of the maximal Abelian gauge, $D_{\mu}^{a b} A_{\mu}^{b}=0$, is attained in the limit $\alpha \rightarrow 0$, which has to be taken after the removal of the ultraviolet divergences. We also remark that the whole term $S_{\alpha}$ vanishes in the limit $\alpha \rightarrow 0$, allowing us to integrate out the localizing fields $\left(\phi_{\mu}^{a b}, \bar{\phi}_{\mu}^{a b}, \omega_{\mu}^{a b}, \bar{\omega}_{\mu}^{a b}\right)$, and thus recovering the horizon function (1).

\subsection{The global $U(8)$ symmetry}

In addition to the BRST invariance the action $S_{2}$ displays a global $U(8)[10,13]$ symmetry expressed by

$$
\mathcal{Q}_{\mu \nu}^{a b} S_{2}=0,
$$

with

$$
\mathcal{Q}_{\mu \nu}^{a b}=\int d^{4} x\left(\phi_{\mu}^{c a} \frac{\delta}{\delta \phi_{\nu}^{c b}}-\bar{\phi}_{\nu}^{c b} \frac{\delta}{\delta \bar{\phi}_{\mu}^{c a}}+\omega_{\mu}^{c a} \frac{\delta}{\delta \omega_{\nu}^{c b}}-\bar{\omega}_{\nu}^{c b} \frac{\delta}{\delta \bar{\omega}_{\mu}^{c a}}+M_{\sigma \mu}^{c a} \frac{\delta}{\delta M_{\sigma \nu}^{c b}}-\bar{M}_{\sigma \nu}^{c b} \frac{\delta}{\delta \bar{M}_{\sigma \mu}^{c a}}+N_{\sigma \mu}^{c a} \frac{\delta}{\delta N_{\sigma \nu}^{c b}}-\bar{N}_{\sigma \nu}^{c b} \frac{\delta}{\delta \bar{N}_{\sigma \mu}^{c a}}\right) .
$$




\begin{tabular}{lcccccccccccc}
\hline \hline & $A$ & $b$ & $\bar{c}$ & $c$ & $\phi$ & $\bar{\phi}$ & $\omega$ & $\bar{\omega}$ & $M$ & $\bar{M}$ & $N$ & $\bar{N}$ \\
\hline $\operatorname{dim}$ & 1 & 2 & 2 & 0 & 1 & 1 & 1 & 1 & 2 & 2 & 2 & 2 \\
gh. number & 0 & 0 & -1 & 1 & 0 & 0 & 1 & -1 & 0 & 0 & 1 & -1 \\
$\mathcal{Q}_{8}$-charge & 0 & 0 & 0 & 0 & 1 & -1 & 1 & -1 & 1 & -1 & 1 & -1 \\
\hline \hline
\end{tabular}

Table 1: Quantum numbers of the fields and sources

The presence of the global invariance $U(8)$ means that one can make use $[10,13]$ of the composite index $i \equiv(a, \mu)$, $i=1, \ldots, 8$. Therefore, setting

$$
\left(\phi_{\mu}^{a b}, \bar{\phi}_{\mu}^{a b}, \omega_{\mu}^{a b}, \bar{\omega}_{\mu}^{a b}\right)=\left(\phi_{i}^{a}, \bar{\phi}_{i}^{a}, \omega_{i}^{a}, \bar{\omega}_{i}^{a}\right),
$$

and

$$
\left(M_{\mu \nu}^{a b}, \bar{M}_{\mu \nu}^{a b}, N_{\mu \nu}^{a b}, \bar{N}_{\mu \nu}^{a b}\right)=\left(M_{\mu i}^{a}, \bar{M}_{\mu i}^{a}, N_{\mu i}^{a}, \bar{N}_{\mu i}^{a}\right),
$$

we can write $S_{2}$ as

$$
\begin{aligned}
S_{2}= & S_{\mathrm{YM}}+S_{\mathrm{MAG}}+\int d^{4} x\left\{\bar{\phi}_{i}^{a} \mathcal{M}^{a b} \phi_{i}^{b}-\bar{\omega}_{i}^{a} \mathcal{M}^{a b} \omega_{i}^{b}+\bar{\omega}_{i}^{a} \mathcal{F}^{a b} \phi_{i}^{b}+\bar{M}_{\mu i}^{a} D_{\mu}^{a b} \phi_{i}^{b}+N_{\mu i}^{a} D_{\mu}^{a b} \bar{\omega}_{i}^{b}\right. \\
& \left.+\bar{N}_{\mu i}^{a}\left[D_{\mu}^{a b} \omega_{i}^{b}+g \varepsilon^{a b}\left(\partial_{\mu} c+g \varepsilon^{c d} A_{\mu}^{c} c^{d}\right) \phi_{i}^{b}\right]+M_{\mu i}^{a}\left[D_{\mu}^{a b} \bar{\phi}_{i}^{b}+g \varepsilon^{a b}\left(\partial_{\mu} c+g \varepsilon^{c d} A_{\mu}^{c} c^{d}\right) \bar{\omega}_{i}^{b}\right]\right\} \\
& +\frac{\alpha}{2} \int d^{4} x\left[b^{a} b^{a}+2 i g \varepsilon^{a b} b^{a} \bar{c}^{b} c-g^{2}\left(\bar{\phi}_{i}^{a} \phi_{i}^{a}-\bar{\omega}_{i}^{a} \omega_{i}^{a}-\bar{c}^{a} c^{a}\right)\left(\bar{\phi}_{j}^{b} \phi_{j}^{b}-\bar{\omega}_{j}^{b} \omega_{j}^{b}-\bar{c}^{b} c^{b}\right)\right. \\
& \left.-2 i g^{2} \bar{\omega}_{i}^{a} \phi_{i}^{a} b^{b} c^{b}+2 g^{3} \bar{\omega}_{i}^{a} \phi_{i}^{a} \varepsilon^{b c} \bar{c}^{b} c^{c} c\right] .
\end{aligned}
$$

For the symmetry generator we have

$$
\mathcal{Q}_{i j}=\int d^{4} x\left(\phi_{i}^{a} \frac{\delta}{\delta \phi_{j}^{a}}-\bar{\phi}_{j}^{a} \frac{\delta}{\delta \bar{\phi}_{i}^{a}}+\omega_{i}^{a} \frac{\delta}{\delta \omega_{j}^{a}}-\bar{\omega}_{j}^{a} \frac{\delta}{\delta \bar{\omega}_{i}^{a}}+M_{\mu i}^{a} \frac{\delta}{\delta M_{\mu j}^{a}}-\bar{M}_{\mu j}^{a} \frac{\delta}{\delta \bar{M}_{\mu i}^{a}}+N_{\mu i}^{a} \frac{\delta}{\delta N_{\mu j}^{a}}-\bar{N}_{\mu j}^{a} \frac{\delta}{\delta \bar{N}_{\mu i}^{a}}\right) .
$$

By means of the trace of the operator $\mathcal{Q}_{i j}$, i.e., $\mathcal{Q}_{i i} \equiv \mathcal{Q}_{8}$, the $i$-valued fields turn out to possess an additional quantum number, displayed in the Table 1, together with the mass dimension and the ghost number.

\subsection{Introduction of external sources}

In order to establish the set of Ward identities, we have first to properly define the nonlinear transformations of the fields, as given in (20). To this purpose, we notice that the BRST transformation of the gauge field $A_{\mu}^{a}$ can be written as the sum of two composite operators, namely

$$
s A_{\mu}^{a}=\mathcal{O}_{1}+\mathcal{O}_{2}
$$

where,

$$
\mathcal{O}_{1}=-D_{\mu}^{a b} c^{b}, \quad \mathcal{O}_{2}=-g \varepsilon^{a b} A_{\mu}^{b} c .
$$

Thanks to the fact that the BRST operator is nilpotent, i.e. $s^{2}=0$, it follows that

$$
s \mathcal{O}_{1}=-s \mathcal{O}_{2} .
$$

These two operators can be defined by means of a suitable set of external sources, $\left(\Omega_{\mu}^{a}, K_{\mu}^{a}, \xi_{\mu}^{a}\right)$, as

$$
S_{\mathrm{ext}}^{(1)}=\int d^{4} x\left[\Omega_{\mu}^{a}\left(-D_{\mu}^{a b} c^{b}\right)+K_{\mu}^{a}\left(-g \varepsilon^{a b} A_{\mu} c\right)+\xi_{\mu}^{a} s\left(-g \varepsilon^{a b} A_{\mu} c\right)\right] .
$$

To guarantee the BRST invariance of $S_{\text {ext }}^{(1)}$ we require that

$$
s \xi_{\mu}^{a}=-\left(\Omega_{\mu}^{a}-K_{\mu}^{a}\right), \quad s \Omega_{\mu}^{a}=s K_{\mu}^{a}=0 .
$$


The nonlinear BRST transformations of the fields $A_{\mu}, c^{a}, c$ can be accounted for by the external sources $\Omega_{\mu}, L^{a}, L$, according to

$$
\begin{aligned}
S_{\mathrm{ext}}^{(2)} & =s \int d^{4} x\left[-\Omega_{\mu} A_{\mu}+L^{a} c^{a}+L c\right] \\
& =\int d^{4} x\left[-\Omega_{\mu}\left(\partial_{\mu} c+g \varepsilon^{a b} A_{\mu}^{a} c^{b}\right)+g \varepsilon^{a b} L^{a} c^{b} c+\frac{g}{2} \varepsilon^{a b} L c^{a} c^{b}\right],
\end{aligned}
$$

where we require that

$$
s \Omega_{\mu}=0, \quad s L^{a}=0, \quad s L=0 .
$$

Moreover, adding $S_{\text {ext }}^{(1)}$ to $S_{2}$ we obtain an action that is left invariant by the following transformations:

The $\delta_{i}$ symmetry:

$$
\delta_{i} \bar{c}^{a}=\phi_{i}^{a},
$$$$
\delta_{i} \bar{\phi}_{j}^{a}=\delta_{i j} c^{a},
$$$$
\delta_{i} b^{a}=-i g \varepsilon^{a b} \phi_{i}^{b} c
$$$$
\delta_{i} \Omega_{\mu}^{a}=M_{\mu i}^{a},
$$

The $\bar{\delta}_{i}$ symmetry:

$$
\begin{aligned}
\bar{\delta}_{i} \bar{c}^{a} & =\bar{\omega}_{i}^{a}, \\
\bar{\delta}_{i} \omega_{j}^{a} & =-\delta_{i j} c^{a}, \\
\bar{\delta}_{i} b^{a} & =-i g \varepsilon^{a b} \bar{\omega}_{i}^{b} c, \\
\bar{\delta}_{i} \Omega_{\mu}^{a} & =-\bar{N}_{\mu i}^{a} .
\end{aligned}
$$

As transformations (55) contain composite field operators, i.e., $g \varepsilon^{a b} \phi_{i}^{b} c$ and $g \varepsilon^{a b} \bar{\omega}^{b} c$, we define them by means of additional external sources $\left(Y_{i}^{a}, X_{i}^{a}\right)$ and $\left(\bar{X}_{i}^{a}, \bar{Y}_{i}^{a}\right)$, giving rise to two sets of BRST doublets

$$
\begin{array}{ll}
s Y_{i}^{a}=X_{i}^{a}, & s X_{i}^{a}=0, \\
s \bar{X}_{i}^{a}=-\bar{Y}_{i}^{a}, & s \bar{Y}_{i}^{a}=0,
\end{array}
$$

so that

$$
\begin{aligned}
S_{\mathrm{ext}}^{(3)}= & -s \int d^{4} x g \varepsilon^{a b}\left(\bar{X}_{i}^{a} \phi_{i}^{b} c-Y_{i}^{a} \bar{\omega}_{i}^{b} c\right) \\
= & \int d^{4} x\left[g \varepsilon^{a b} \bar{Y}_{i}^{a} \phi_{i}^{b} c-\bar{X}_{i}^{a}\left(g \varepsilon^{a b} \omega_{i}^{b} c+\frac{g^{2}}{2} \varepsilon^{a b} \varepsilon^{c d} \phi_{i}^{b} c^{c} c^{d}\right)\right. \\
& \left.+g \varepsilon^{a b} X_{i}^{a} \bar{\omega}_{i}^{b} c-Y_{i}^{a}\left(g \varepsilon^{a b} \bar{\phi}_{i}^{b} c-\frac{g^{2}}{2} \varepsilon^{a b} \varepsilon^{c d} \bar{\omega}_{i}^{b} c^{c} c^{d}\right)\right] .
\end{aligned}
$$

Therefore, for the most general invariant external source term which can be added to $S_{2}$, we obtain

$$
S_{\mathrm{ext}}=S_{\mathrm{ext}}^{(1)}+S_{\mathrm{ext}}^{(2)}+S_{\mathrm{ext}}^{(3)}+\chi \int d^{4} x\left(\bar{M}_{\mu i}^{a} M_{\mu i}^{a}+\bar{N}_{\mu i}^{a} N_{\mu i}^{a}\right)
$$

where the last term, which can be written as an exact BRST variation

$$
\chi \int d^{4} x\left(\bar{M}_{\mu i}^{a} M_{\mu i}^{a}+\bar{N}_{\mu i}^{a} N_{\mu i}^{a}\right)=-\chi s \int d^{4} x \bar{N}_{\mu i}^{a} M_{\mu i}^{a},
$$

is allowed by power counting and has to be added for renormalization purposes. Also, the parameter $\chi$ stands for a free coefficient.

\subsection{Introduction of dimension two operators}

The last step towards the construction of the complete starting classical action is the introduction of the three dimension two operators $\mathcal{O}_{A^{2}}, \mathcal{O}_{\bar{f} f}$ and $\mathcal{O}_{\text {ghost }}$, eqs.(7),(9),(13). Let us start by considering the gluon operator $\mathcal{O}_{A^{2}}(x)=\frac{1}{2} A_{\mu}^{a}(x) A_{\mu}^{a}(x)$. Introducing the BRST doublet of sources $(\lambda, J)$ as

$$
s \lambda=J, \quad s J=0,
$$

it turns out that $\mathcal{O}_{A^{2}}$ can be introduced in a BRST invariant way, namely

$$
S_{J}=s \int d^{4} x \lambda\left(\mathcal{O}_{A^{2}}+\frac{1}{2} \zeta J\right)=\int d^{4} x\left(J \mathcal{O}_{A^{2}}+\frac{1}{2} \zeta J^{2}+\lambda A_{\mu}^{a} D_{\mu}^{a b} c^{b}\right),
$$


where $\zeta$ is a constant parameter needed to account for the ultraviolet divergences of the vacuum correlation function $\left\langle\left(A_{\mu}^{a}(x) A_{\mu}^{a}(x)\right)\left(A_{\nu}^{b}(y) A_{\nu}^{b}(y)\right)\right\rangle$.

The operators $\mathcal{O}_{\bar{f} f}, \mathcal{O}_{\text {ghost }}$ can be introduced in a similar way. More specifically, defining the BRST doublet of sources $(\tau, \sigma)$ as

$$
s \tau=\sigma, \quad s \sigma=0,
$$

the invariant term $S_{\sigma}$ describing the coupling of $\mathcal{O}_{\bar{f} f}$ is given by

$$
S_{\sigma}=s \int d^{4} x \tau\left(\mathcal{O}_{\bar{f} f}+\frac{1}{2} \kappa \sigma+\rho J\right)=\int d^{4} x\left(\sigma \mathcal{O}_{\bar{f} f}+\frac{1}{2} \kappa \sigma^{2}+\rho \sigma J-\tau s \mathcal{O}_{\bar{f} f}\right),
$$

where $\kappa$ and $\rho$ are constant parameters, needed for renormalization purposes. Notice in fact that expression (63) contains the mixing term $\sigma J$. This term, allowed by power counting, accounts for the ultraviolet divergences of the mixed vacuum correlation function $\left\langle\left(A_{\mu}^{a}(x) A_{\mu}^{a}(x)\right)\left(\bar{\phi}_{i}^{b}(y) \phi_{i}^{b}(y)-\bar{\omega}_{i}^{b}(y) \omega_{i}^{b}(y)-\bar{c}^{b}(y) c^{b}(y)\right)\right\rangle$.

Finally, the introduction of a third doublet of sources $(\eta, \theta)$

$$
s \eta=\theta, \quad s \theta=0,
$$

allows us to introduce the ghost operator $\mathcal{O}_{\text {ghost }}(x)=g \varepsilon^{a b} \bar{c}^{a}(x) c^{b}(x)$, namely

$$
S_{\theta}=s \int d^{4} x \eta\left(\mathcal{O}_{\text {ghost }}+\frac{1}{2} \beta \theta\right)=\int d^{4} x\left(\theta \mathcal{O}_{\text {ghost }}+\frac{1}{2} \beta \theta^{2}-\eta s \mathcal{O}_{\text {ghost }}\right),
$$

where $\beta$ is a constant parameter needed for the divergences of the correlation function $\left\langle\left(\varepsilon^{a b} \bar{c}^{a}(x) c^{b}(x)\right)\left(\varepsilon^{m n} \bar{c}^{m}(y) c^{n}(y)\right)\right\rangle$. Notice, however, that the ghost operator $\mathcal{O}_{\text {ghost }}$ breaks the symmetries (55). Therefore, to maintain the symmetry content of the theory is necessary to introduce two more BRST doubles of external sources,

$$
\begin{array}{ll}
s \eta_{i}=-\theta_{i}, & s \theta_{i}=0, \\
s \bar{\theta}_{i}=\bar{\eta}_{i}, & s \bar{\eta}_{i}=0,
\end{array}
$$

and define an extra term given by

$$
\begin{aligned}
S_{\text {extra }} & =s \int d^{4} x g \varepsilon^{a b}\left(\bar{\theta}_{i} \phi_{i}^{a} c^{b}-\eta_{i} \bar{\omega}_{i}^{a} c^{b}\right) \\
& =\int d^{4} x\left[g \varepsilon^{a b}\left(\bar{\eta}_{i} \phi_{i}^{a} c^{b}+\eta_{i} \bar{\phi}_{i}^{a} c^{b}+\bar{\theta}_{i} \omega_{i}^{a} c^{b}+\theta_{i} \bar{\omega}_{i}^{a} c^{b}\right)-g^{2} \bar{\theta}_{i} \phi_{i}^{a} c^{a} c+g^{2} \eta_{i} \bar{\omega}_{i}^{a} c^{a} c\right] .
\end{aligned}
$$

\subsection{The complete classical action}

We are now ready to write down the complete classical action $\Sigma$, given by

$$
\begin{aligned}
\Sigma & =\Sigma_{0}+S_{\text {extra }}, \\
\Sigma_{0} & =S_{2}+S_{\text {ext }}+S_{J}+S_{\sigma}+S_{\theta}, \\
S_{2} & =S_{1}+S_{\alpha}, \\
S_{1} & =S_{0}+S_{\mathrm{Local}}^{\text {inv }}, \\
S_{0} & =S_{\mathrm{YM}}+S_{\mathrm{MAG}},
\end{aligned}
$$

where, $S_{\mathrm{YM}}, S_{\mathrm{MAG}}, S_{\mathrm{Local}}^{\text {inv }}, S_{\alpha}, S_{\mathrm{ext}}, S_{J}, S_{\sigma}, S_{\theta}, S_{\text {extra }}$ are given, respectively, by (16), (17), (30), (39), (58), (61), (63), (65), (67). Thus, the complete classical action is

$$
\Sigma=S_{\mathrm{YM}}+S_{\mathrm{MAG}}+S_{\mathrm{Local}}^{\mathrm{inv}}+S_{\alpha}+S_{\mathrm{ext}}+S_{J}+S_{\sigma}+S_{\theta}+S_{\mathrm{extra}}
$$


or, explicitly, we have

$$
\begin{aligned}
& \Sigma=S_{\mathrm{YM}}+s \int d^{4} x\left(\bar{c}^{a} D_{\mu}^{a b} A_{\mu}^{b}+\bar{c} \partial_{\mu} A_{\mu}+\bar{\omega}_{i}^{a} \mathcal{M}^{a b} \phi_{i}^{b}-\bar{N}_{\mu i}^{a} D_{\mu}^{a b} \phi_{i}^{b}+M_{\mu i}^{a} D_{\mu}^{a b} \bar{\omega}_{i}^{b}\right. \\
& \left.-g \varepsilon^{a b} \bar{X}_{i}^{a} \phi_{i}^{b} c+g \varepsilon^{a b} Y_{i}^{a} \bar{\omega}_{i}^{b} c-\Omega_{\mu}^{a} A_{\mu}^{a}-g \varepsilon^{a b} \xi_{\mu}^{a} A_{\mu}^{b} c-\Omega_{\mu} A_{\mu}+L^{a} c^{a}+L c-\chi \bar{N}_{\mu i}^{a} M_{\mu i}^{a}\right) \\
& -\frac{\alpha}{2} s \int d^{4} x\left[\bar{c}^{a} i b^{a}-g \varepsilon^{a b} \bar{c}^{a} \bar{c}^{b} c+g^{2} \bar{\omega}_{i}^{a} \phi_{i}^{a}\left(\bar{\phi}_{j}^{b} \phi_{j}^{b}-\bar{\omega}_{j}^{b} \omega_{j}^{b}\right)-2 g^{2} \bar{\omega}_{i}^{a} \phi_{i}^{a} \bar{c}^{b} c^{b}\right] \\
& +s \int d^{4} x \lambda\left(\frac{1}{2} A_{\mu}^{a} A_{\mu}^{a}+\frac{1}{2} \zeta J\right)+s \int d^{4} x \tau\left[\left(\bar{\phi}_{i}^{a} \phi_{i}^{a}-\bar{\omega}_{i}^{a} \omega_{i}^{a}-\bar{c}^{a} c^{a}\right)+\frac{1}{2} \kappa \sigma+\rho J\right] \\
& +s \int d^{4} x\left[g \varepsilon^{a b}\left(\eta \bar{c}^{a} c^{b}+\bar{\theta}_{i} \phi_{i}^{a} c^{b}-\eta_{i} \bar{\omega}_{i}^{a} c^{b}\right)+\frac{1}{2} \beta \eta \theta\right] \\
& =S_{\mathrm{YM}}+\int d^{4} x\left\{i b^{a} D_{\mu}^{a b} A_{\mu}^{b}-\bar{c}^{a} \mathcal{M}^{a b} c^{b}+g \varepsilon^{a b} \bar{c}^{a}\left(D_{\mu}^{b c} A_{\mu}^{c}\right) c+i b \partial_{\mu} A_{\mu}+\bar{c} \partial_{\mu}\left(\partial_{\mu} c+g \varepsilon^{a b} A_{\mu}^{a} c^{b}\right)+\bar{\phi}_{i}^{a} \mathcal{M}^{a b} \phi_{i}^{b}\right. \\
& -\bar{\omega}_{i}^{a} \mathcal{M}^{a b} \omega_{i}^{b}+\bar{\omega}_{i}^{a} \mathcal{F}^{a b} \phi_{i}^{b}+\bar{M}_{\mu i}^{a} D_{\mu}^{a b} \phi_{i}^{b}+\bar{N}_{\mu i}^{a}\left[D_{\mu}^{a b} \omega_{i}^{b}+g \varepsilon^{a b}\left(\partial_{\mu} c+g \varepsilon^{c d} A_{\mu}^{c} c^{d}\right) \phi_{i}^{b}\right]+N_{\mu i}^{a} D_{\mu}^{a b} \bar{\omega}_{i}^{b} \\
& +M_{\mu i}^{a}\left[D_{\mu}^{a b} \bar{\phi}_{i}^{b}+g \varepsilon^{a b}\left(\partial_{\mu} c+g \varepsilon^{c d} A_{\mu}^{c} c^{d}\right) \bar{\omega}_{i}^{b}\right]-\Omega_{\mu}^{a} D_{\mu}^{a b} c^{b}-g \varepsilon^{a b} K_{\mu}^{a} A_{\mu}^{b} c+\xi_{\mu}^{a}\left[g \varepsilon^{a b}\left(D_{\mu}^{b c} c^{c}\right) c\right. \\
& \left.-\frac{g^{2}}{2} \varepsilon^{a b} \varepsilon^{c d} A_{\mu}^{b} c^{c} c^{d}\right]-\Omega_{\mu}\left(\partial_{\mu} c+g \varepsilon^{a b} A_{\mu}^{a} c^{b}\right)+g \varepsilon^{a b} L^{a} c^{b} c+\frac{g}{2} \varepsilon^{a b} L c^{a} c^{b}+g \varepsilon^{a b} \bar{Y}_{i}^{a} \phi_{i}^{b} c-\bar{X}_{i}^{a}\left[g \varepsilon^{a b} \omega_{i}^{b} c\right. \\
& \left.+\frac{g^{2}}{2} \varepsilon^{a b} \varepsilon^{c d} \phi_{i}^{b} c^{c} c^{d}\right]+g \varepsilon^{a b} X_{i}^{a} \bar{\omega}_{i}^{b} c-Y_{i}^{a}\left[g \varepsilon^{a b} \bar{\phi}_{i}^{b} c-\frac{g^{2}}{2} \varepsilon^{a b} \varepsilon^{c d} \bar{\omega}_{i}^{b} c^{c} c^{d}\right]+\chi\left(\bar{M}_{\mu i}^{a} M_{\mu i}^{a}+\bar{N}_{\mu i}^{a} N_{\mu i}^{a}\right) \\
& +\frac{\alpha}{2}\left[b^{a} b^{a}+2 i g \varepsilon^{a b} b^{a} \bar{c}^{b} c-g^{2}\left(\bar{\phi}_{i}^{a} \phi_{i}^{a}-\bar{\omega}_{i}^{a} \omega_{i}^{a}-\bar{c}^{a} c^{a}\right)\left(\bar{\phi}_{j}^{b} \phi_{j}^{b}-\bar{\omega}_{j}^{b} \omega_{j}^{b}-\bar{c}^{b} c^{b}\right)-2 i g^{2} \bar{\omega}_{i}^{a} \phi_{i}^{a} b^{b} c^{b}\right. \\
& \left.+2 g^{3} \bar{\omega}_{i}^{a} \phi_{i}^{a} \varepsilon^{b c} \bar{c}^{b} c^{c} c\right]+\frac{1}{2} J A_{\mu}^{a} A_{\mu}^{a}+\lambda A_{\mu}^{a} D_{\mu}^{a b} c^{b}+\sigma\left(\bar{\phi}_{i}^{a} \phi_{i}^{a}-\bar{\omega}_{i}^{a} \omega_{i}^{a}-\bar{c}^{a} c^{a}\right)+\tau\left(i b^{a} c^{a}-g \varepsilon^{a b} \bar{c}^{a} c^{b} c\right) \\
& +\frac{\zeta}{2} J^{2}+\rho J \sigma+\frac{\kappa}{2} \sigma^{2}+g \varepsilon^{a b}\left(\theta \bar{c}^{a} c^{b}+\bar{\eta}_{i} \phi_{i}^{a} c^{b}+\eta_{i} \bar{\phi}_{i}^{a} c^{b}+\bar{\theta}_{i} \omega_{i}^{a} c^{b}+\theta_{i} \bar{\omega}_{i}^{a} c^{b}\right)-i g \varepsilon^{a b} \eta b^{a} c^{b}-g^{2} \eta \bar{c}^{a} c^{a} c \\
& \left.-g^{2} \bar{\theta}_{i} \phi_{i}^{a} c^{a} c+g^{2} \eta_{i} \bar{\omega}_{i}^{a} c^{a} c+\frac{\beta}{2} \theta^{2}\right\} \text {. }
\end{aligned}
$$

The expressions for the gluon and ghost propagators given in Sect.2 are easily derived by considering the relevant quadratic terms of $(70)$ and by replacing $(J, \sigma, \theta)$ by the more conventional mass parameters $\left(m^{2}, \mu^{2}, v^{2}\right)$ originating from the corresponding dimension two condensates, i.e. $m^{2} \sim\left\langle A_{\mu}^{a} A_{\mu}^{a}\right\rangle[8], \mu^{2} \sim\left\langle\left(\bar{\phi}_{i}^{a} \phi_{i}^{a}-\bar{\omega}_{i}^{a} \omega_{i}^{a}-\bar{c}^{a} c^{a}\right)\right\rangle[32]$, $v^{2} \sim\left\langle\varepsilon^{a b} \bar{c}^{a} c^{b}\right\rangle[14]$

The action (70) constitutes our starting point in order to establish the renormalizability of the model.

\section{$4 \quad$ Ward identities}

It turns out that $\Sigma$ fulfills the following set of Ward identities:

- The Slavnov-Taylor identity:

$$
\begin{aligned}
\mathcal{S}(\Sigma) \equiv & \int d^{4} x\left[\left(\frac{\delta \Sigma}{\delta \Omega_{\mu}^{a}}+\frac{\delta \Sigma}{\delta K_{\mu}^{a}}\right) \frac{\delta \Sigma}{\delta A_{\mu}^{a}}+\frac{\delta \Sigma}{\delta \Omega_{\mu}} \frac{\delta \Sigma}{\delta A_{\mu}}+\frac{\delta \Sigma}{\delta L^{a}} \frac{\delta \Sigma}{\delta c^{a}}+\frac{\delta \Sigma}{\delta L} \frac{\delta \Sigma}{\delta c}+i b^{a} \frac{\delta \Sigma}{\delta \bar{c}^{a}}+i b \frac{\delta \Sigma}{\delta \bar{c}}\right. \\
& +\omega_{i}^{a} \frac{\delta \Sigma}{\delta \phi_{i}^{a}}+\bar{\phi}_{i}^{a} \frac{\delta \Sigma}{\delta \bar{\omega}_{i}^{a}}+N_{\mu i}^{a} \frac{\delta \Sigma}{\delta M_{\mu i}^{a}}-\bar{M}_{\mu i}^{a} \frac{\delta \Sigma}{\delta \bar{N}_{\mu i}^{a}}-\left(\Omega_{\mu}^{a}-K_{\mu}^{a}\right) \frac{\delta \Sigma}{\delta \xi_{\mu}^{a}}-\bar{Y}_{i}^{a} \frac{\delta \Sigma}{\delta \bar{X}_{i}^{a}} \\
& \left.+X_{i}^{a} \frac{\delta \Sigma}{\delta Y_{i}^{a}}+J \frac{\delta \Sigma}{\delta \lambda}+\sigma \frac{\delta \Sigma}{\delta \tau}+\theta \frac{\delta \Sigma}{\delta \eta}-\theta_{i} \frac{\delta \Sigma}{\delta \eta_{i}}+\bar{\eta}_{i} \frac{\delta \Sigma}{\delta \bar{\theta}_{i}}\right]=0 .
\end{aligned}
$$

- The four global $\mathcal{W}_{i}^{(N)}$-identities which mix the Faddev-Popov ghost fields with the auxiliary localizing fields:

$$
\mathcal{W}_{i}^{(N)}(\Sigma)=0, \quad(N=1,2,3,4),
$$




\begin{tabular}{lcccccccccccccccccc}
\hline \hline & $\Omega$ & $K$ & $\xi$ & $L$ & $Y$ & $\bar{Y}$ & $X$ & $\bar{X}$ & $\lambda$ & $\tau$ & $J$ & $\sigma$ & $\eta$ & $\theta$ & $\eta_{i}$ & $\bar{\eta}_{i}$ & $\theta_{i}$ & $\bar{\theta}_{i}$ \\
\hline $\operatorname{dim}$ & 3 & 3 & 3 & 4 & 3 & 3 & 3 & 3 & 2 & 2 & 2 & 2 & 2 & 2 & 3 & 3 & 3 & 3 \\
gh. number & -1 & -1 & -2 & -2 & -1 & -1 & 0 & -2 & -1 & -1 & 0 & 0 & -1 & 0 & -1 & -1 & 0 & -2 \\
$\mathcal{Q}_{8}$-charge & 0 & 0 & 0 & 0 & 1 & -1 & 1 & -1 & 0 & 0 & 0 & 0 & 0 & 0 & 1 & -1 & 1 & -1 \\
\hline \hline
\end{tabular}

Table 2: Quantum numbers of the external sources

where

$$
\begin{aligned}
& \mathcal{W}_{i}^{(1)}(\Sigma) \equiv \int d^{4} x\left(\phi_{i}^{a} \frac{\delta \Sigma}{\delta \bar{c}^{a}}+c^{a} \frac{\delta \Sigma}{\delta \bar{\phi}_{i}^{a}}+M_{\mu i}^{a} \frac{\delta \Sigma}{\delta \Omega_{\mu}^{a}}-Y_{i}^{a} \frac{\delta \Sigma}{\delta L^{a}}-i \frac{\delta \Sigma}{\delta \bar{Y}_{i}^{a}} \frac{\delta \Sigma}{\delta b^{a}}-\theta \frac{\delta \Sigma}{\delta \bar{\eta}_{i}}+2 \eta_{i} \frac{\delta \Sigma}{\delta L}\right) \\
& \mathcal{W}_{i}^{(2)}(\Sigma) \equiv \int d^{4} x\left(\bar{\omega}_{i}^{a} \frac{\delta \Sigma}{\delta \bar{c}^{a}}-c^{a} \frac{\delta \Sigma}{\delta \omega_{i}^{a}}-\bar{N}_{\mu i}^{a} \frac{\delta \Sigma}{\delta \Omega_{\mu}^{a}}-\bar{X}_{i}^{a} \frac{\delta \Sigma}{\delta L^{a}}-i \frac{\delta \Sigma}{\delta X_{i}^{a}} \frac{\delta \Sigma}{\delta b^{a}}-\theta \frac{\delta \Sigma}{\delta \theta_{i}}+2 \bar{\theta}_{i} \frac{\delta \Sigma}{\delta L}\right), \\
& \mathcal{W}_{i}^{(3)}(\Sigma) \equiv \int d^{4} x\left[\left(\frac{\delta \Sigma}{\delta \bar{Y}_{i}^{a}}+\omega_{i}^{a}\right) \frac{\delta \Sigma}{\delta \bar{c}^{a}}+i \frac{\delta \Sigma}{\delta \bar{X}_{i}^{a}} \frac{\delta \Sigma}{\delta b^{a}}+\left(\frac{\delta \Sigma}{\delta \bar{\phi}_{i}^{a}}-X_{i}^{a}\right) \frac{\delta \Sigma}{\delta L^{a}}+c^{a} \frac{\delta \Sigma}{\delta \bar{\omega}_{i}^{a}}\right. \\
& \left.-M_{\mu i}^{a} \frac{\delta \Sigma}{\delta \xi_{\mu}^{a}}+N_{\mu i}^{a} \frac{\delta \Sigma}{\delta \Omega_{\mu}^{a}}-\theta \frac{\delta \Sigma}{\delta \bar{\theta}_{i}}-2 \theta_{i} \frac{\delta \Sigma}{\delta L}\right] \\
& \mathcal{W}_{i}^{(4)}(\Sigma) \equiv \int d^{4} x\left[\left(\frac{\delta \Sigma}{\delta X_{i}^{a}}-\bar{\phi}_{i}^{a}\right) \frac{\delta \Sigma}{\delta \bar{c}^{a}}-i \frac{\delta \Sigma}{\delta Y_{i}^{a}} \frac{\delta \Sigma}{\delta b^{a}}+\left(\frac{\delta \Sigma}{\delta \omega_{i}^{a}}-\bar{Y}_{i}^{a}\right) \frac{\delta \Sigma}{\delta L^{a}}-c^{a} \frac{\delta \Sigma}{\delta \phi_{i}^{a}}\right. \\
& \left.+\bar{N}_{\mu i}^{a} \frac{\delta \Sigma}{\delta \xi_{\mu}^{a}}-\bar{M}_{\mu i}^{a} \frac{\delta \Sigma}{\delta \Omega_{\mu}^{a}}+\theta \frac{\delta \Sigma}{\delta \eta_{i}}-2 \bar{\eta}_{i} \frac{\delta \Sigma}{\delta L}\right] .
\end{aligned}
$$

- The global $U(8)$ invariance:

$$
\mathcal{Q}_{i j}(\Sigma)=0
$$

with

$$
\begin{aligned}
\mathcal{Q}_{i j}= & \int d^{4} x\left(\phi_{i}^{a} \frac{\delta}{\delta \phi_{j}^{a}}-\bar{\phi}_{j}^{a} \frac{\delta}{\delta \bar{\phi}_{i}^{a}}+\omega_{i}^{a} \frac{\delta}{\delta \omega_{j}^{a}}-\bar{\omega}_{j}^{a} \frac{\delta}{\delta \bar{\omega}_{i}^{a}}+M_{\mu i}^{a} \frac{\delta}{\delta M_{\mu j}^{a}}-\bar{M}_{\mu j}^{a} \frac{\delta}{\delta \bar{M}_{\mu i}^{a}}+N_{\mu i}^{a} \frac{\delta}{\delta N_{\mu j}^{a}}\right. \\
& \left.-\bar{N}_{\mu j}^{a} \frac{\delta}{\delta \bar{N}_{\mu i}^{a}}+Y_{i}^{a} \frac{\delta}{\delta Y_{j}^{a}}-\bar{Y}_{j}^{a} \frac{\delta}{\delta \bar{Y}_{i}^{a}}+X_{i}^{a} \frac{\delta}{\delta X_{j}^{a}}-\bar{X}_{j}^{a} \frac{\delta}{\delta \bar{X}_{i}^{a}}+\eta_{i} \frac{\delta}{\delta \eta_{j}}-\bar{\eta}_{j} \frac{\delta}{\delta \bar{\eta}_{i}}+\theta_{i} \frac{\delta}{\delta \theta_{j}}-\bar{\theta}_{j} \frac{\delta}{\delta \bar{\theta}_{i}}\right) .
\end{aligned}
$$

The trace of (75) defines a new charge displayed in the Tables 1 and 2. This operator generalizes that of eq. (47).

- The exact rigid symmetries:

$$
\begin{aligned}
\mathfrak{R}_{i j}(\Sigma) \equiv & \int d^{4} x\left(\phi_{i}^{a} \frac{\delta \Sigma}{\delta \omega_{j}^{a}}-\bar{\omega}_{j}^{a} \frac{\delta \Sigma}{\delta \bar{\phi}_{i}^{a}}+M_{\mu i}^{a} \frac{\delta \Sigma}{\delta N_{\mu j}^{a}}+\bar{N}_{\mu j}^{a} \frac{\delta \Sigma}{\delta \bar{M}_{\mu i}^{a}}+Y_{i}^{a} \frac{\delta \Sigma}{\delta X_{j}^{a}}+\bar{X}_{j}^{a} \frac{\delta \Sigma}{\delta \bar{Y}_{i}^{a}}\right. \\
& \left.-\eta_{i} \frac{\delta \Sigma}{\delta \theta_{j}}-\bar{\theta}_{j} \frac{\delta \Sigma}{\delta \bar{\eta}_{i}}\right)=0, \\
\mathfrak{R}^{(1)}(\Sigma) \equiv & \int d^{4} x\left(\bar{\omega}_{i}^{a} \frac{\delta \Sigma}{\delta \omega_{i}^{a}}-\bar{N}_{\mu i}^{a} \frac{\delta \Sigma}{\delta N_{\mu i}^{a}}+\bar{X}_{i}^{a} \frac{\delta \Sigma}{\delta X_{i}^{a}}-\bar{\theta}_{i} \frac{\delta \Sigma}{\delta \theta_{i}}\right)=0, \\
\mathfrak{R}^{(2)}(\Sigma) \equiv & \int d^{4} x\left(\bar{\omega}_{i}^{a} \frac{\delta \Sigma}{\delta \phi_{i}^{a}}-\bar{\phi}_{i}^{a} \frac{\delta \Sigma}{\delta \omega_{i}^{a}}-\bar{N}_{\mu i}^{a} \frac{\delta \Sigma}{\delta M_{\mu i}^{a}}-\bar{M}_{\mu i}^{a} \frac{\delta \Sigma}{\delta N_{\mu i}^{a}}+\bar{X}_{i}^{a} \frac{\delta \Sigma}{\delta Y_{i}^{a}}+\bar{Y}_{i}^{a} \frac{\delta \Sigma}{\delta X_{i}^{a}}\right. \\
& \left.+\bar{\theta}_{i} \frac{\delta \Sigma}{\delta \eta_{i}}+\bar{\eta}_{i} \frac{\delta \Sigma}{\delta \theta_{i}}\right)=0 .
\end{aligned}
$$

- The diagonal gauge fixing condition:

$$
\frac{\delta \Sigma}{\delta b}=i \partial_{\mu} A_{\mu}
$$


- The diagonal anti-ghost equation:

$$
\frac{\delta \Sigma}{\delta \bar{c}}+\partial_{\mu} \frac{\delta \Sigma}{\delta \Omega_{\mu}}=0
$$

- The $S L(2, \mathbb{R})$ symmetry:

$$
\mathcal{D}(\Sigma) \equiv \int d^{4} x\left(c^{a} \frac{\delta \Sigma}{\delta \bar{c}^{a}}-i \frac{\delta \Sigma}{\delta L^{a}} \frac{\delta \Sigma}{\delta b^{a}}-2 \theta \frac{\delta \Sigma}{\delta L}\right)=0
$$

- The local $U(1)$ invariance:

$$
\mathcal{W}^{3}(\Sigma)=-i \partial^{2} b
$$

with

$$
\mathcal{W}^{3} \equiv \partial_{\mu} \frac{\delta}{\delta A_{\mu}}+g \varepsilon^{a b} \sum_{\mathcal{Y} \in \text { Off }} \mathcal{Y}^{a} \frac{\delta}{\delta \mathcal{Y}^{b}}
$$

where

$$
\text { Off }=\left\{A_{\mu}^{a}, b^{a}, \bar{c}^{a}, c^{a}, \bar{\phi}_{i}^{a}, \phi_{i}^{a}, \bar{\omega}_{i}^{a}, \omega_{i}^{a}, \Omega_{\mu}^{a}, K_{\mu}^{a}, \xi_{\mu}^{a}, L^{a}, \bar{X}_{i}^{a}, X_{i}^{a}, \bar{Y}_{i}^{a}, Y_{i}^{a}, \bar{M}_{\mu i}^{a}, M_{\mu i}^{a}, \bar{N}_{\mu i}^{a}, N_{\mu i}^{a}\right\}
$$

- The BRST on-shell invariance of the general operator $\left(\mathcal{O}_{A^{2}}+\alpha \mathcal{O}_{\overline{f f}}\right)$ :

$$
\mathcal{U}(\Sigma) \equiv \int d^{4} x\left(\frac{\delta \Sigma}{\delta \lambda}+\alpha \frac{\delta \Sigma}{\delta \tau}-i c^{a} \frac{\delta \Sigma}{\delta b^{a}}-2 \eta \frac{\delta \Sigma}{\delta L}\right)=0 .
$$

\section{Algebraic characterization of the most general counterterm}

We can face now the issue of the renormalizability of the starting action $\Sigma$. We shall employ the algebraic renormalization [38] and look for the most general invariant counterterm which can be freely added to all orders of perturbation theory. To that purpose we perturb the classical action $\Sigma$ by adding an arbitrary integrated local polynomial $\Sigma_{\mathrm{CT}}$ in the fields and external sources of dimension bounded by four, zero ghost number and zero $\mathcal{Q}_{8}$-charge. Requiring thus the perturbed action, $\Sigma+\epsilon \Sigma_{\mathrm{CT}}$, satisfies the same Ward identities as $\Sigma$ to the first order in the perturbation parameter $\epsilon$, we get:

$$
\begin{aligned}
\mathcal{S}\left(\Sigma+\epsilon \Sigma_{\mathrm{CT}}\right) & =0+O\left(\epsilon^{2}\right) \\
\frac{\delta}{\delta b}\left(\Sigma+\epsilon \Sigma_{\mathrm{CT}}\right) & =i \partial_{\mu} A_{\mu}+O\left(\epsilon^{2}\right) \\
\left(\frac{\delta}{\delta \bar{c}}+\partial_{\mu} \frac{\delta}{\delta \Omega_{\mu}}\right)\left(\Sigma+\epsilon \Sigma_{\mathrm{CT}}\right) & =0+O\left(\epsilon^{2}\right) \\
\mathcal{W}_{i}^{(N)}\left(\Sigma+\epsilon \Sigma_{\mathrm{CT}}\right) & =0+O\left(\epsilon^{2}\right), \quad(N=1,2,3,4), \quad(K=1,2) \\
\mathfrak{R}_{i j}\left(\Sigma+\epsilon \Sigma_{\mathrm{CT}}\right) & =0+O\left(\epsilon^{2}\right), \\
\mathfrak{R}^{(K)}\left(\Sigma+\epsilon \Sigma_{\mathrm{CT}}\right) & =0+O\left(\epsilon^{2}\right), \\
\mathcal{Q}_{i j}\left(\Sigma+\epsilon \Sigma_{\mathrm{CT}}\right) & =0+O\left(\epsilon^{2}\right) \\
\mathcal{D}\left(\Sigma+\epsilon \Sigma_{\mathrm{CT}}\right) & =0+O\left(\epsilon^{2}\right) \\
\mathcal{W}^{3}\left(\Sigma+\epsilon \Sigma_{\mathrm{CT}}\right) & =-i \partial^{2} b+O\left(\epsilon^{2}\right) \\
\mathcal{U}\left(\Sigma+\epsilon \Sigma_{\mathrm{CT}}\right) & =0+O\left(\epsilon^{2}\right) .
\end{aligned}
$$


This amounts to imposing the following conditions on $\Sigma_{\mathrm{CT}}$

$$
\begin{aligned}
\mathcal{S}_{\Sigma} \Sigma_{\mathrm{CT}} & =0, \\
\frac{\delta}{\delta b} \Sigma_{\mathrm{CT}} & =0, \\
\left(\frac{\delta}{\delta \bar{c}}+\partial_{\mu} \frac{\delta}{\delta \Omega_{\mu}}\right) \Sigma_{\mathrm{CT}} & =0, \\
\mathcal{W}_{i}^{\Sigma(N)} \Sigma_{\mathrm{CT}} & =0, \quad(N=1,2,3,4), \\
\mathfrak{R}_{i j} \Sigma_{\mathrm{CT}} & =0, \\
\mathfrak{R}^{(K)} \Sigma_{\mathrm{CT}} & =0, \quad(K=1,2), \\
\mathcal{Q}_{i j} \Sigma_{\mathrm{CT}} & =0, \\
\mathcal{D}_{\Sigma} \Sigma_{\mathrm{CT}} & =0, \\
\mathcal{W}^{3} \Sigma_{\mathrm{CT}} & =0, \\
\mathcal{U} \Sigma_{\mathrm{CT}} & =0,
\end{aligned}
$$

where $\mathcal{S}_{\Sigma}$ is the nilpotent linearized Slavnov-Taylor operator,

$$
\begin{aligned}
& \mathcal{S}_{\Sigma} \mathcal{S}_{\Sigma}=0 \\
& \mathcal{S}_{\Sigma}= \int d^{4} x\left[\left(\frac{\delta \Sigma}{\delta \Omega_{\mu}^{a}}+\frac{\delta \Sigma}{\delta K_{\mu}^{a}}\right) \frac{\delta}{\delta A_{\mu}^{a}}+\frac{\delta \Sigma}{\delta A_{\mu}^{a}}\left(\frac{\delta}{\delta \Omega_{\mu}^{a}}+\frac{\delta}{\delta K_{\mu}^{a}}\right)+\frac{\delta \Sigma}{\delta \Omega_{\mu}} \frac{\delta}{\delta A_{\mu}}+\frac{\delta \Sigma}{\delta A_{\mu}} \frac{\delta}{\delta \Omega_{\mu}}+\frac{\delta \Sigma}{\delta L^{a}} \frac{\delta}{\delta c^{a}}\right. \\
&+ \frac{\delta \Sigma}{\delta c^{a}} \frac{\delta}{\delta L^{a}}+\frac{\delta \Sigma}{\delta L} \frac{\delta}{\delta c}+\frac{\delta \Sigma}{\delta c} \frac{\delta}{\delta L}+i b^{a} \frac{\delta}{\delta \bar{c}^{a}}+i b \frac{\delta}{\delta \bar{c}}+\omega_{i}^{a} \frac{\delta}{\delta \phi_{i}^{a}}+\bar{\phi}_{i}^{a} \frac{\delta}{\delta \bar{\omega}_{i}^{a}}+N_{\mu i}^{a} \frac{\delta}{\delta M_{\mu i}^{a}}-\bar{M}_{\mu i}^{a} \frac{\delta}{\delta \bar{N}_{\mu i}^{a}} \\
&\left.-\left(\Omega_{\mu}^{a}-K_{\mu}^{a}\right) \frac{\delta}{\delta \xi_{\mu}^{a}}-\bar{Y}_{i}^{a} \frac{\delta}{\delta \bar{X}_{i}^{a}}+X_{i}^{a} \frac{\delta}{\delta Y_{i}^{a}}+J \frac{\delta}{\delta \lambda}+\sigma \frac{\delta}{\delta \tau}+\theta \frac{\delta}{\delta \eta}-\theta_{i} \frac{\delta}{\delta \eta_{i}}+\bar{\eta}_{i} \frac{\delta}{\delta \bar{\theta}_{i}}\right],
\end{aligned}
$$

while $\mathcal{W}_{i}^{\Sigma(N)}$, with $N=1, \ldots, 4$, and $\mathcal{D}_{\Sigma}$ are the linearized operators corresponding to the Ward identities $(73)$ and (79), respectively, and they are given by

$$
\begin{aligned}
\mathcal{W}_{i}^{\Sigma(1)}= & \int d^{4} x\left(\phi_{i}^{a} \frac{\delta}{\delta \bar{c}^{a}}+c^{a} \frac{\delta}{\delta \bar{\phi}_{i}^{a}}+M_{\mu i}^{a} \frac{\delta}{\delta \Omega_{\mu}^{a}}-Y_{i}^{a} \frac{\delta}{\delta L^{a}}-i \frac{\delta \Sigma}{\delta \bar{Y}_{i}^{a}} \frac{\delta}{\delta b^{a}}-i \frac{\delta \Sigma}{\delta b^{a}} \frac{\delta}{\delta \bar{Y}_{i}^{a}}-\theta \frac{\delta}{\delta \bar{\eta}_{i}}+2 \eta_{i} \frac{\delta}{\delta L}\right), \\
\mathcal{W}_{i}^{\Sigma(2)}= & \int d^{4} x\left(\bar{\omega}_{i}^{a} \frac{\delta}{\delta \bar{c}^{a}}-c^{a} \frac{\delta}{\delta \omega_{i}^{a}}-\bar{N}_{\mu i}^{a} \frac{\delta}{\delta \Omega_{\mu}^{a}}-\bar{X}_{i}^{a} \frac{\delta}{\delta L^{a}}-i \frac{\delta \Sigma}{\delta X_{i}^{a}} \frac{\delta}{\delta b^{a}}-i \frac{\delta \Sigma}{\delta b^{a}} \frac{\delta}{\delta X_{i}^{a}}-\theta \frac{\delta}{\delta \theta_{i}}+2 \bar{\theta}_{i} \frac{\delta}{\delta L}\right), \\
\mathcal{W}_{i}^{\Sigma(3)}= & \int d^{4} x\left[\left(\frac{\delta \Sigma}{\delta \bar{Y}_{i}^{a}}+\omega_{i}^{a}\right) \frac{\delta}{\delta \bar{c}^{a}}+\frac{\delta \Sigma}{\delta \bar{c}^{a}} \frac{\delta}{\delta \bar{Y}_{i}^{a}}+i \frac{\delta \Sigma}{\delta \bar{X}_{i}^{a}} \frac{\delta}{\delta b^{a}}+i \frac{\delta \Sigma}{\delta b^{a}} \frac{\delta}{\delta \bar{X}_{i}^{a}}+\left(\frac{\delta \Sigma}{\delta \bar{\phi}_{i}^{a}}-X_{i}^{a}\right) \frac{\delta}{\delta L^{a}}\right. \\
& \left.+\frac{\delta \Sigma}{\delta L^{a}} \frac{\delta}{\delta \bar{\phi}_{i}^{a}}+c^{a} \frac{\delta}{\delta \bar{\omega}_{i}^{a}}-M_{\mu i}^{a} \frac{\delta}{\delta \xi_{\mu}^{a}}+N_{\mu i}^{a} \frac{\delta}{\delta \Omega_{\mu}^{a}}-\theta \frac{\delta}{\delta \bar{\theta}_{i}}-2 \theta_{i} \frac{\delta}{\delta L}\right], \\
\mathcal{W}_{i}^{\Sigma(4)}= & \int d^{4} x\left[\left(\frac{\delta \Sigma}{\delta X_{i}^{a}}-\bar{\phi}_{i}^{a}\right) \frac{\delta}{\delta \bar{c}^{a}}+\frac{\delta \Sigma}{\delta \bar{c}^{a}} \frac{\delta}{\delta X_{i}^{a}}-i \frac{\delta \Sigma}{\delta Y_{i}^{a}} \frac{\delta}{\delta b^{a}}-i \frac{\delta \Sigma}{\delta b^{a}} \frac{\delta}{\delta Y_{i}^{a}}+\left(\frac{\delta \Sigma}{\delta \omega_{i}^{a}}-\bar{Y}_{i}^{a}\right) \frac{\delta}{\delta L^{a}}\right. \\
& \left.+\frac{\delta \Sigma}{\delta L^{a}} \frac{\delta}{\delta \omega_{i}^{a}}-c^{a} \frac{\delta}{\delta \phi_{i}^{a}}+\bar{N}_{\mu i}^{a} \frac{\delta}{\delta \xi_{\mu}^{a}}-\bar{M}_{\mu i}^{a} \frac{\delta}{\delta \Omega_{\mu}^{a}}+\theta \frac{\delta}{\delta \eta_{i}}-2 \bar{\eta}_{i} \frac{\delta}{\delta L}\right],
\end{aligned}
$$

and

$$
\mathcal{D}_{\Sigma}=\int d^{4} x\left(c^{a} \frac{\delta}{\delta \bar{c}^{a}}-i \frac{\delta \Sigma}{\delta L^{a}} \frac{\delta}{\delta b^{a}}-i \frac{\delta \Sigma}{\delta b^{a}} \frac{\delta}{\delta L^{a}}-2 \theta \frac{\delta}{\delta L}\right) .
$$

For further use, let us write some useful commutation and anticommutation relations

$$
\begin{aligned}
\left\{\mathcal{W}_{i}^{\Sigma(1)}, \mathcal{S}_{\Sigma}\right\} & =\mathcal{W}_{i}^{\Sigma(3)}, & {\left[\mathcal{W}_{i}^{\Sigma(2)}, \mathcal{S}_{\Sigma}\right] } & =\mathcal{W}_{i}^{\Sigma(4)} \\
\left\{\mathfrak{R}_{i j}, \mathcal{S}_{\Sigma}\right\} & =\mathcal{Q}_{i j}, & {\left[\mathfrak{R}^{(1)}, \mathcal{S}_{\Sigma}\right] } & =\mathfrak{R}^{(2)} \\
{\left[\mathcal{W}^{3}, \mathcal{S}_{\Sigma}\right] } & =0, & \left\{\mathcal{U}, \mathcal{S}_{\Sigma}\right\} & =\mathcal{D}_{\Sigma} .
\end{aligned}
$$

From the second and the third constraints of (85) it follows that $\Sigma_{\mathrm{CT}}$ is independent from the diagonal Lagrange multiplier $b$, and that the diagonal antighost $\bar{c}$ enters only through the combination $\left(\Omega_{\mu}+\partial_{\mu} \bar{c}\right)$. Furthermore, 
from general results on the cohomology of gauge theories [38], it turns out that the most general solution of the constraint $\mathcal{S}_{\Sigma} \Sigma_{\mathrm{CT}}=0$, i.e. the first of eqs.(85), can be written as

$$
\Sigma_{\mathrm{CT}}=a_{0} S_{\mathrm{YM}}+\mathcal{S}_{\Sigma} \Delta^{(-1)}
$$

with $\Delta^{(-1)}$ being an integrated local polynomial with ghost number -1 , given by

$$
\begin{aligned}
& \Delta^{(-1)}=\int d^{4} x\left[a_{1} \Omega_{\mu}^{a} A_{\mu}^{a}+a_{2} K_{\mu}^{a} A_{\mu}^{a}+a_{3} \xi_{\mu}^{a} g \varepsilon^{a b} A_{\mu}^{b} c+a_{4} \xi_{\mu}^{a} \partial_{\mu} c^{a}+a_{5} \xi_{\mu}^{a} g \varepsilon^{a b} A_{\mu} c^{b}+a_{6}\left(\partial_{\mu} \bar{c}^{a}\right) A_{\mu}^{a}\right. \\
& +a_{7}\left(\Omega_{\mu}+\partial_{\mu} \bar{c}\right) A_{\mu}+a_{8} c^{a} L^{a}+a_{9} c L+a_{10} \bar{X}_{i}^{a} g \varepsilon^{a b} \phi_{i}^{b} c+a_{11} \bar{X}_{i}^{a} \omega_{i}^{a}+a_{12} Y_{i}^{a} g \varepsilon^{a b} \bar{\omega}_{i}^{b} c \\
& +a_{13} Y_{i}^{a} \bar{\phi}_{i}^{a}+a_{14} \bar{Y}_{i}^{a} \phi_{i}^{a}+a_{15} X_{i}^{a} \bar{\omega}_{i}^{a}+a_{16} \bar{N}_{\mu i}^{a} \partial_{\mu} \phi_{i}^{a}+a_{17} \bar{N}_{\mu i}^{a} g \varepsilon^{a b} A_{\mu} \phi_{i}^{b}+a_{18} M_{\mu i}^{a} \partial_{\mu} \bar{\omega}_{i}^{a} \\
& +a_{19} M_{\mu i}^{a} g \varepsilon^{a b} A_{\mu} \bar{\omega}_{i}^{b}+a_{20} i b^{a} \bar{c}^{a}+a_{21} g \varepsilon^{a b} \bar{c}^{a} \bar{c}^{b} c+a_{22} g \varepsilon^{a b} \bar{c}^{a} A_{\mu} A_{\mu}^{b}+a_{23} \bar{\omega}_{i}^{a} \phi_{i}^{a} \bar{\phi}_{j}^{b} \phi_{j}^{b} \\
& +a_{24} \bar{\omega}_{i}^{a} \phi_{i}^{a} \bar{\omega}_{j}^{b} \omega_{j}^{b}+a_{25} \bar{\omega}_{i}^{a} \phi_{i}^{b} \bar{\phi}_{j}^{a} \phi_{j}^{b}+a_{26} \bar{\omega}_{i}^{a} \phi_{i}^{b} \bar{\omega}_{j}^{a} \omega_{j}^{b}+a_{27} \bar{\omega}_{i}^{a} \phi_{i}^{b} \bar{\phi}_{j}^{b} \phi_{j}^{a}+a_{28} \bar{\omega}_{i}^{a} \phi_{i}^{b} \bar{\omega}_{j}^{b} \omega_{j}^{a} \\
& +a_{29} \bar{\omega}_{i}^{a} \phi_{j}^{a} \bar{\phi}_{i}^{b} \phi_{j}^{b}+a_{30} \bar{\omega}_{i}^{a} \phi_{j}^{b} \bar{\phi}_{i}^{a} \phi_{j}^{b}+a_{31} \bar{\omega}_{i}^{a} \phi_{j}^{a} \bar{\omega}_{i}^{b} \omega_{j}^{b}+a_{32} \bar{\omega}_{i}^{a} \phi_{i}^{a} \bar{c}^{b} c^{b}+a_{33} \bar{\omega}_{i}^{a} \phi_{i}^{b} \bar{c}^{a} c^{b} \\
& +a_{34} \bar{\omega}_{i}^{a} \phi_{i}^{b} \bar{c}^{b} c^{a}+a_{35} \bar{\omega}_{i}^{a} \phi_{i}^{a} A_{\mu} A_{\mu}+a_{36} \bar{\omega}_{i}^{a} \phi_{i}^{a} A_{\mu}^{b} A_{\mu}^{b}+a_{37} \bar{\omega}_{i}^{a} \phi_{i}^{b} A_{\mu}^{a} A_{\mu}^{b}+a_{38} \bar{\omega}_{i}^{a} \partial^{2} \phi_{i}^{a} \\
& +a_{39} \bar{\omega}_{i}^{a} g \varepsilon^{a b} A_{\mu} \partial_{\mu} \phi_{i}^{b}+a_{40} \bar{\omega}_{i}^{a} g \varepsilon^{a b}\left(\partial_{\mu} A_{\mu}\right) \phi_{i}^{b}+a_{41} \chi \bar{N}_{\mu i}^{a} M_{\mu i}^{a}+\frac{1}{2}\left(a_{42} \lambda+a_{43} \tau\right) A_{\mu}^{a} A_{\mu}^{a} \\
& +\frac{1}{2}\left(a_{44} \lambda+a_{45} \tau\right) A_{\mu} A_{\mu}+\left(a_{46} \lambda+a_{47} \tau\right) \bar{c}^{a} c^{a}+\left(a_{48} \lambda+a_{49} \tau\right) \bar{\phi}_{i}^{a} \phi_{i}^{a}+\left(a_{50} \lambda+a_{51} \tau\right) \bar{\omega}_{i}^{a} \omega_{i}^{a} \\
& +\left(a_{52} J+a_{53} \sigma\right) \bar{\omega}_{i}^{a} \phi_{i}^{a}+\left(a_{54} J+a_{55} \sigma\right) \lambda+\left(a_{56} J+a_{57} \sigma\right) \tau+a_{58} \eta \partial_{\mu} A_{\mu}+a_{59} \eta \theta \\
& \left.+g \varepsilon^{a b}\left(a_{60} \eta \bar{c}^{a} c^{b}+a_{61} \eta \bar{\phi}_{i}^{a} \phi_{i}^{b}+a_{62} \eta \bar{\omega}_{i}^{a} \omega_{i}^{b}+a_{63} \theta \bar{\omega}_{i}^{a} \phi_{i}^{b}+a_{64} \bar{\theta}_{i} \phi_{i}^{a} c^{b}+a_{65} \eta_{i} \bar{\omega}_{i}^{a} c^{b}\right)\right],
\end{aligned}
$$

where the coefficients $a_{n}, n=0, \ldots, 65$, are free dimensionless parameters. Notice also that in the derivation of expression (92) use has been made of the fact that the action $\Sigma$, and thus $\Sigma_{\mathrm{CT}}$, are left invariant by the following discrete symmetry

$$
\mathcal{Y}^{1} \rightarrow \mathcal{Y}^{1}, \quad \mathcal{Y}^{2} \rightarrow-\mathcal{Y}^{2}, \quad \mathcal{Y}^{\text {diag }} \rightarrow-\mathcal{Y}^{\text {diag }}, \quad \mathcal{Y} \rightarrow \mathcal{Y}
$$

where $\mathcal{Y}^{a}$, with $a=1,2$, stands for the elements of the off-diagonal set (82), while $\mathcal{Y}^{\text {diag }}$ for the diagonal sector

$$
\mathcal{Y}^{\text {diag }} \in\left\{A_{\mu}, b, c, \bar{c}, \Omega_{\mu}, L, \eta, \theta, \eta_{i}, \bar{\eta}_{i}, \theta_{i}, \bar{\theta}_{i}\right\},
$$

and $\mathcal{Y}$ the sources $\lambda, \tau, J, \sigma$. As one can easily recognize, this symmetry plays the role of the charge conjugation.

After a quite lengthy calculation one finds that the most general expression for $\Delta^{(-1)}$ compatible with all constraints (85) and the discrete symmetry (93) is

$$
\begin{aligned}
\Delta^{(-1)}= & \int d^{4} x\left\{\left(a_{1}+a_{2}\right)\left(\Omega_{\mu}^{a} A_{\mu}^{a}+g \varepsilon^{a b} \xi_{\mu}^{a} A_{\mu}^{b} c\right)+\left(a_{2}+a_{4}\right) \xi_{\mu}^{a} D_{\mu}^{a b} c^{b}+\left(a_{1}-a_{4}+a_{8}\right)\left(\bar{N}_{\mu i}^{a} D_{\mu}^{a b} \phi_{i}^{b}-M_{\mu i}^{a} D_{\mu}^{a b} \bar{\omega}_{i}^{b}\right)\right. \\
& -\left(a_{6}+a_{8}\right) \bar{\omega}_{i}^{a} \mathcal{M}^{a b} \phi_{i}^{b}-a_{6} \bar{c}^{a} D_{\mu}^{a b} A_{\mu}^{b}+a_{8} L^{a} c^{a}+a_{9} L c+a_{41} \chi \bar{N}_{\mu i}^{a} M_{\mu i}^{a}+a_{20}\left(i \bar{c}^{a} b^{a}-g \varepsilon^{a b} \bar{c}^{a} \bar{c}^{b} c\right) \\
& -\left(-\alpha a_{8}-a_{20}\right) g^{2} \bar{\omega}_{i}^{a} \phi_{i}^{a}\left(\bar{\phi}_{j}^{b} \phi_{j}^{b}-\bar{\omega}_{j}^{b} \omega_{j}^{b}\right)+\left(-\alpha a_{8}-2 a_{20}\right) g^{2} \bar{\omega}_{i}^{a} \phi_{i}^{a} \bar{c}^{b} c^{b}+\frac{1}{2}\left(a_{8}-a_{6}-\alpha a_{43}\right) \lambda A_{\mu}^{a} A_{\mu}^{a} \\
& +\frac{1}{2} a_{43} \tau A_{\mu}^{a} A_{\mu}^{a}-\left(-\alpha a_{8}+2 a_{20}-\alpha a_{47}\right) \lambda\left(\bar{\phi}_{i}^{a} \phi_{i}^{a}-\bar{\omega}_{i}^{a} \omega_{i}^{a}-\bar{c}^{a} c^{a}\right)-\left(a_{8}+a_{47}\right) \tau\left(\bar{\phi}_{i}^{a} \phi_{i}^{a}-\bar{\omega}_{i}^{a} \omega_{i}^{a}\right)+a_{47} \tau \bar{c}^{a} c^{a} \\
& \left.-\left(a_{8}-a_{9}\right) g \varepsilon^{a b}\left(\eta \bar{c}^{a} c^{b}+\bar{\theta}_{i} \phi_{i}^{a} c^{b}-\eta_{i} \bar{\omega}^{a} c^{b}\right)+a_{54} J \lambda+a_{55} \sigma \lambda+a_{56} J \tau+a_{57} \sigma \tau+a_{59} \eta \theta\right\} .
\end{aligned}
$$

Noticing that

$$
J \tau=\sigma \lambda+\mathcal{S}_{\Sigma}(\lambda \tau)
$$

and renaming the coefficients as

$$
\begin{gathered}
a_{1}+a_{2} \rightarrow a_{1}, \quad a_{2}+a_{4} \rightarrow-a_{2}, \quad a_{8} \rightarrow a_{3}, \\
a_{6} \rightarrow a_{4}, \quad a_{9} \rightarrow a_{5}, \quad a_{41} \rightarrow a_{6}, \quad a_{20} \rightarrow-\frac{1}{2} \alpha a_{7}, \\
a_{43} \rightarrow a_{8}, \quad a_{47} \rightarrow a_{9}, \quad a_{54} \rightarrow a_{10} \frac{1}{2} \zeta, \\
a_{55}+a_{56} \rightarrow a_{11} \rho, \quad a_{57} \rightarrow a_{12} \frac{1}{2} \kappa, \quad a_{59} \rightarrow a_{13} \frac{1}{2} \beta,
\end{gathered}
$$


we get

$$
\begin{aligned}
\Delta^{(-1)}= & \int d^{4} x\left\{a_{1}\left(\Omega_{\mu}^{a} A_{\mu}^{a}+g \varepsilon^{a b} \xi_{\mu}^{a} A_{\mu}^{b} c\right)-a_{2} \xi_{\mu}^{a} D_{\mu}^{a b} c^{b}+\left(a_{1}+a_{2}+a_{3}\right)\left(\bar{N}_{\mu i}^{a} D_{\mu}^{a b} \phi_{i}^{b}-M_{\mu i}^{a} D_{\mu}^{a b} \bar{\omega}_{i}^{b}\right)\right. \\
& +a_{3} L^{a} c^{a}-\left(a_{3}+a_{4}\right) \bar{\omega}_{i}^{a} \mathcal{M}^{a b} \phi_{i}^{b}-a_{4} \bar{c}^{a} D_{\mu}^{a b} A_{\mu}^{b}+a_{5} L c+a_{6} \chi \bar{N}_{\mu i}^{a} M_{\mu i}^{a}-\frac{\alpha}{2} a_{7}\left(i \bar{c}^{a} b^{a}-g \varepsilon^{a b} \bar{c}^{a} \bar{c}^{b} c\right) \\
& -\frac{\alpha}{2}\left(a_{7}-2 a_{3}\right) g^{2} \bar{\omega}_{i}^{a} \phi_{i}^{a}\left(\bar{\phi}_{j}^{b} \phi_{j}^{b}-\bar{\omega}_{j}^{b} \omega_{j}^{b}\right)+\alpha\left(a_{7}-a_{3}\right) g^{2} \bar{\omega}_{i}^{a} \phi_{i}^{a} \bar{c}^{b} c^{b}+\frac{1}{2}\left(a_{3}-a_{4}-\alpha a_{8}\right) \lambda A_{\mu}^{a} A_{\mu}^{a} \\
& +\frac{1}{2} a_{8} \tau A_{\mu}^{a} A_{\mu}^{a}+\alpha\left(a_{3}+a_{7}+a_{9}\right) \lambda\left(\bar{\phi}_{i}^{a} \phi_{i}^{a}-\bar{\omega}_{i}^{a} \omega_{i}^{a}-\bar{c}^{a} c^{a}\right)-\left(a_{3}+a_{9}\right) \tau\left(\bar{\phi}_{i}^{a} \phi_{i}^{a}-\bar{\omega}_{i}^{a} \omega_{i}^{a}\right)+a_{9} \tau \bar{c}^{a} c^{a} \\
& \left.-\left(a_{3}-a_{5}\right) g \varepsilon^{a b}\left(\eta \bar{c}^{a} c^{b}+\bar{\theta}_{i} \phi_{i}^{a} c^{b}-\eta_{i} \bar{\omega}^{a} c^{b}\right)+a_{10} \frac{1}{2} \zeta J \lambda+a_{11} \rho \sigma \lambda+a_{12} \frac{1}{2} \kappa \sigma \tau+a_{13} \frac{1}{2} \beta \eta \theta\right\},
\end{aligned}
$$

so that for the counterterm $\Sigma_{\mathrm{CT}}$ we get

$$
\begin{aligned}
& \Sigma_{\mathrm{CT}}=\int d^{4} x\left\{( a _ { 0 } + 2 a _ { 1 } ) \left[\frac{1}{2}\left(\partial_{\mu} A_{\nu}^{a}\right)\left(\partial_{\mu} A_{\nu}^{a}-\partial_{\nu} A_{\mu}^{a}\right)-g \varepsilon^{a b}\left(\partial_{\mu} A_{\nu}^{a}\right)\left(A_{\mu} A_{\nu}^{b}-A_{\nu} A_{\mu}^{b}\right)+g \varepsilon^{a b}\left(\partial_{\mu} A_{\nu}\right) A_{\mu}^{a} A_{\nu}^{b}\right.\right. \\
& \left.+\frac{g^{2}}{2}\left(A_{\mu} A_{\mu} A_{\nu}^{a} A_{\nu}^{a}+A_{\mu} A_{\nu} A_{\mu}^{a} A_{\nu}^{a}\right)\right]+\frac{a_{0}}{2}\left(\partial_{\mu} A_{\nu}\right)\left(\partial_{\mu} A_{\nu}-\partial_{\nu} A_{\mu}\right)+\left(a_{0}+4 a_{1}\right) \frac{g^{2}}{4} A_{\mu}^{a} A_{\mu}^{a} A_{\nu}^{b} A_{\nu}^{b} \\
& +i\left(a_{1}-a_{4}\right) b^{a} D_{\mu}^{a b} A_{\mu}^{b}-\left(a_{3}+a_{4}\right)\left[\bar{c}^{a} \partial^{2} c^{a}-\bar{c}^{a} g \varepsilon^{a b}\left(\partial_{\mu} A_{\mu}\right) c^{b}-2 \bar{c}^{a} g \varepsilon^{a b} A_{\mu} \partial_{\mu} c^{b}-g^{2} \bar{c}^{a} c^{a} A_{\mu} A_{\mu}\right] \\
& +\left(a_{3}+a_{4}\right)\left[\bar{\phi}_{i}^{a} \partial^{2} \phi_{i}^{a}-\bar{\phi}_{i}^{a} g \varepsilon^{a b}\left(\partial_{\mu} A_{\mu}\right) \phi_{i}^{b}-2 \bar{\phi}_{i}^{a} g \varepsilon^{a b} A_{\mu} \partial_{\mu} \phi_{i}^{b}-g^{2} \bar{\phi}_{i}^{a} \phi_{i}^{a} A_{\mu} A_{\mu}\right] \\
& -\left(a_{3}+a_{4}\right)\left[\bar{\omega}_{i}^{a} \partial^{2} \omega_{i}^{a}-\bar{\omega}_{i}^{a} g \varepsilon^{a b}\left(\partial_{\mu} A_{\mu}\right) \omega_{i}^{b}-2 \bar{\omega}_{i}^{a} g \varepsilon^{a b} A_{\mu} \partial_{\mu} \omega_{i}^{b}-g^{2} \bar{\omega}_{i}^{a} \omega_{i}^{a} A_{\mu} A_{\mu}\right] \\
& +\left(2 a_{1}-a_{3}-a_{4}\right) g^{2} \varepsilon^{a c} \varepsilon^{b d}\left(\bar{c}^{a} c^{b}-\bar{\phi}_{i}^{a} \phi_{i}^{b}+\bar{\omega}_{i}^{a} \omega_{i}^{b}\right) A_{\mu}^{c} A_{\mu}^{d}+\left(a_{1}-a_{4}-a_{5}\right) g \varepsilon^{a b} \bar{c}^{a} c D_{\mu}^{b c} A_{\mu}^{c} \\
& -\left(a_{1}-a_{3}\right)\left(\Omega_{\mu}+\partial_{\mu} \bar{c}\right) g \varepsilon^{a b} A_{\mu}^{a} c^{b}+\left(a_{1}-2 a_{3}-a_{4}\right)\left[2 g^{2} \varepsilon^{a b} \varepsilon^{c d} \bar{\omega}_{i}^{a} A_{\mu}^{c} c^{d} \partial_{\mu} \phi_{i}^{b}+g^{2} \varepsilon^{a b} \varepsilon^{c d} \bar{\omega}_{i}^{a} \partial\left(A_{\mu}^{c} c^{d}\right) \phi_{i}^{b}\right. \\
& \left.+2 g^{3} \varepsilon^{b c} \bar{\omega}_{i}^{a} \phi_{i}^{a} A_{\mu} A_{\mu}^{b} c^{c}-g^{2}\left(\varepsilon^{a c} \varepsilon^{b d}+\varepsilon^{a d} \varepsilon^{b c}\right) \bar{\omega}_{i}^{a} A_{\mu}^{d}\left(D_{\mu}^{c e} c^{e}\right) \phi_{i}^{b}\right]+\left(2 a_{1}-a_{3}-a_{4}-a_{5}\right) g^{3}\left(\delta^{a e} \varepsilon^{b d}+\delta^{b e} \varepsilon^{a d}\right) \\
& \times \bar{\omega}_{i}^{a} A_{\mu}^{d} A_{\mu}^{e} \phi_{i}^{b} c-\left(a_{2}+2 a_{3}\right) g^{2} \varepsilon^{a b} \varepsilon^{c d}\left(\bar{N}_{\mu i}^{a} \phi_{i}^{b}+M_{\mu i}^{a} \bar{\omega}_{i}^{b}\right) A_{\mu}^{c} c^{d}+\left(a_{1}+a_{2}+a_{3}\right) \Omega_{\mu}^{a} D_{\mu}^{a b} c^{b}-a_{2} K_{\mu}^{a} D_{\mu}^{a b} c^{b} \\
& +a_{5} g \varepsilon^{a b} K_{\mu}^{a} A_{\mu}^{b} c-\left(a_{1}+a_{2}+a_{3}+a_{5}\right) g \varepsilon^{a b} \xi_{\mu}^{a}\left(D_{\mu}^{b c} c^{c}\right) c+\left(a_{2}+2 a_{3}\right) \frac{g^{2}}{2} \varepsilon^{a b} \varepsilon^{c d} \xi_{\mu}^{a} A_{\mu}^{b} c^{c} c^{d}-a_{5} g \varepsilon^{a b} L^{a} c^{b} c \\
& -\left(2 a_{3}-a_{5}\right) \frac{g^{2}}{2} \varepsilon^{a b} L c^{a} c^{b}+a_{5}\left(\Omega_{\mu}+\partial_{\mu} \bar{c}\right) \partial_{\mu} c+a_{3} g^{2} \varepsilon^{a b} \varepsilon^{c d}\left(\bar{X}_{i}^{a} \phi_{i}^{b}-Y_{i}^{a} \bar{\omega}_{i}^{b}\right) c^{c} c^{d}-a_{5} g \varepsilon^{a b}\left(\bar{Y}_{i}^{a} \phi_{i}^{b}-\bar{X}_{i}^{a} \omega_{i}^{b}\right. \\
& \left.+X_{i}^{a} \bar{\omega}_{i}^{b}-Y_{i}^{a} \bar{\phi}_{i}^{b}\right) c-\left(a_{3}+a_{4}+a_{5}\right)\left[2 g \varepsilon^{a b} \bar{\omega}_{i}^{a}\left(\partial_{\mu} c\right) \partial_{\mu} \phi_{i}^{b}+g \varepsilon^{a b} \bar{\omega}_{i}^{a}\left(\partial^{2} c\right) \phi_{i}^{b}+2 g^{2} \bar{\omega}_{i}^{a} \phi_{i}^{a} A_{\mu} \partial_{\mu} c\right] \\
& +\left(a_{1}+a_{2}+a_{3}+a_{5}\right) g \varepsilon^{a b}\left(\partial_{\mu} c\right)\left(\bar{N}_{\mu i}^{a} \phi_{i}^{b}-M_{\mu i}^{a} \bar{\omega}_{i}^{b}\right)-\left(a_{1}+a_{2}+a_{3}\right)\left(\bar{M}_{\mu i}^{a} D_{\mu}^{a b} \phi_{i}^{b}+\bar{N}_{\mu i}^{a} D_{\mu}^{a b} \omega_{i}^{b}+M_{\mu i}^{a} D_{\mu}^{a b} \bar{\phi}_{i}^{b}\right. \\
& \left.+N_{\mu i}^{a} D_{\mu}^{a b} \bar{\omega}_{i}^{b}\right)-a_{6} \chi\left(\bar{M}_{\mu i}^{a} M_{\mu i}^{a}+\bar{N}_{\mu i}^{a} N_{\mu i}^{a}\right)+\frac{\alpha}{2}\left[a_{7} b^{a} b^{a}+2 i\left(a_{7}-a_{5}\right) g \varepsilon^{a b} b^{a} \bar{c}^{b} c-g^{2}\left(a_{7}-2 a_{3}\right)\left(\bar{\phi}_{i}^{a} \phi_{i}^{a}\right.\right. \\
& \left.\left.-\bar{\omega}_{i}^{a} \omega_{i}^{a}-\bar{c}^{a} c^{a}\right)\left(\bar{\phi}_{j}^{b} \phi_{j}^{b}-\bar{\omega}_{j}^{b} \omega_{j}^{b}-\bar{c}^{b} c^{b}\right)-2 i g^{2}\left(a_{7}-2 a_{3}\right) \bar{\omega}_{i}^{a} \phi_{i}^{a} b^{b} c^{b}+2 g^{3}\left(a_{7}-2 a_{3}-a_{5}\right) \bar{\omega}_{i}^{a} \phi_{i}^{a} \varepsilon^{b c} \bar{c}^{b} c^{c} c\right] \\
& +\left(2 a_{1}+a_{3}-a_{4}-\alpha a_{8}\right) \frac{1}{2} J A_{\mu}^{a} A_{\mu}^{a}+\left(a_{1}-a_{4}-\alpha a_{8}\right) \lambda A_{\mu}^{a} D_{\mu}^{a b} c^{b}+\left(a_{3}+a_{9}\right)\left(\sigma \bar{c}^{a} c^{a}-i \tau b^{a} c^{a}\right) \\
& +\left(a_{3}+a_{5}+a_{9}\right) \tau g \varepsilon^{a b} \bar{c}^{a} c^{b} c-\left(2 a_{3}-a_{5}\right) g \varepsilon^{a b}\left(\theta \bar{c}^{a} c^{b}+\bar{\eta}_{i} \phi_{i}^{a} c^{b}+\eta_{i} \bar{\phi}_{i}^{a} c^{b}+\bar{\theta}_{i} \omega_{i}^{a} c^{b}+\theta_{i} \bar{\omega}_{i}^{a} c^{b}-i \eta b^{a} c^{b}\right) \\
& +2 g^{2} a_{3}\left(\eta \bar{c}^{a} c^{a} c+\bar{\theta}_{i} \phi_{i}^{a} c^{a} c-\eta_{i} \bar{\omega}_{i}^{a} c^{a} c\right)+a_{8} \frac{1}{2} \sigma A_{\mu}^{a} A_{\mu}^{a}+a_{8} \tau A_{\mu}^{a} D_{\mu}^{a b} c^{b}+\alpha\left(a_{3}+a_{7}+a_{9}\right)\left[J \left(\bar{\phi}_{i}^{a} \phi_{i}^{a}\right.\right. \\
& \left.\left.-\bar{\omega}_{i}^{a} \omega_{i}^{a}-\bar{c}^{a} c^{a}\right)+\lambda\left(i b^{a} c^{a}-g \varepsilon^{a b} \bar{c}^{a} c^{b} c\right)\right]-\left(a_{3}+a_{9}\right) \sigma\left(\bar{\phi}_{i}^{a} \phi_{i}^{a}-\bar{\omega}_{i}^{a} \omega_{i}^{a}\right)+a_{10} \frac{\zeta}{2} J^{2}+a_{11} \rho J \sigma \\
& \left.+a_{12} \frac{\kappa}{2} \sigma^{2}+a_{13} \frac{\beta}{2} \theta^{2}\right\} .
\end{aligned}
$$

After the characterization of the most general local counterterm $\Sigma_{\mathrm{CT}}$ compatible with all constraints, eqs.(85), we still have to check if it can be reabsorbed through a multiplicative redefinition of the fields, sources and parameters of the starting action $\Sigma$, according to

$$
\Sigma\left[\Psi_{0}, \psi_{0}, \mathcal{J}_{0}, \vartheta_{0}, \Omega_{0}, K_{0}, \lambda_{0}, \tau_{0}, J_{0}, \sigma_{0}\right]=\Sigma[\Psi, \psi, \mathcal{J}, \vartheta, \Omega, K, \lambda, \tau, J, \sigma]+\epsilon \Sigma_{\mathrm{CT}}+O\left(\epsilon^{2}\right),
$$

where

$$
\Psi_{0}=\widetilde{Z}_{\Psi}^{1 / 2} \Psi, \quad \psi_{0}=Z_{\psi}^{1 / 2} \psi, \quad \mathcal{J}_{0}=Z_{\mathcal{J}} \mathcal{J}, \quad \vartheta_{0}=Z_{\vartheta} \vartheta
$$


with

$$
\begin{aligned}
\Psi & \equiv\left\{A_{\mu}^{a}, b^{a}, c^{a}, \bar{c}^{a}\right\} \\
\psi & \equiv\left\{A_{\mu}, b, c, \bar{c}, \phi_{i}^{a}, \bar{\phi}_{i}^{a}, \omega_{i}^{a}, \bar{\omega}_{i}^{a}\right\} \\
\mathcal{J} & \equiv\left\{\xi_{\mu}^{a}, L^{a}, L, \Omega_{\mu}, X_{i}^{a}, \bar{X}_{i}^{a}, Y_{i}^{a}, \bar{Y}_{i}^{a}, M_{\mu i}^{a}, \bar{M}_{\mu i}^{a}, N_{\mu i}^{a}, \bar{N}_{\mu i}^{a}, \eta, \theta, \bar{\eta}_{i}, \eta_{i}, \bar{\theta}_{i}, \theta_{i}\right\}, \\
\vartheta & \equiv\{g, \alpha, \chi, \zeta, \rho, \kappa, \beta\} .
\end{aligned}
$$

Moreover, by taking into account the mixing of the sources displaying the same quantum numbers, i.e, $\left(\Omega_{\mu}^{a}, K_{\mu}^{a}\right)$, $(\lambda, \tau)$ and $(J, \sigma)$, we shall set

$$
\left(\begin{array}{l}
\Omega_{0 \mu}^{a} \\
K_{0 \mu}^{a}
\end{array}\right)=\mathbb{Z}_{\Omega K}\left(\begin{array}{c}
\Omega_{\mu}^{a} \\
K_{\mu}^{a}
\end{array}\right), \quad\left(\begin{array}{c}
\lambda_{0} \\
\tau_{0}
\end{array}\right)=\mathbb{Z}_{\lambda \tau}\left(\begin{array}{c}
\lambda \\
\tau
\end{array}\right), \quad\left(\begin{array}{c}
J_{0} \\
\sigma_{0}
\end{array}\right)=\mathbb{Z}_{J \sigma}\left(\begin{array}{c}
J \\
\sigma
\end{array}\right)
$$

where the $\mathbb{Z}$-matrices are given by

$$
\mathbb{Z}_{\Omega K}=\mathbb{I}+\epsilon\left(\begin{array}{cc}
z_{\Omega} & z_{\Omega K} \\
z_{K \Omega} & z_{K}
\end{array}\right), \quad \mathbb{Z}_{\lambda \tau}=\mathbb{I}+\epsilon\left(\begin{array}{cc}
z_{\lambda} & z_{\lambda \tau} \\
z_{\tau \lambda} & z_{\tau}
\end{array}\right), \quad \mathbb{Z}_{J \sigma}=\mathbb{I}+\epsilon\left(\begin{array}{cc}
z_{J} & z_{J \sigma} \\
z_{\sigma J} & z_{\sigma}
\end{array}\right) .
$$

By direct inspection of $\Sigma_{\mathrm{CT}}$, the renormalization factors are found to be

$$
\begin{gathered}
Z_{A}^{1 / 2}=Z_{g}^{-1}, \quad \widetilde{Z}_{\bar{c}}^{1 / 2}=\widetilde{Z}_{c}^{1 / 2}, \\
\widetilde{Z}_{b}^{1 / 2}=Z_{g} Z_{\bar{c}}^{-1 / 2} \widetilde{Z}_{c}^{1 / 2}, \quad Z_{b}^{1 / 2}=Z_{g}, \\
Z_{\phi}^{1 / 2}=Z_{\bar{\phi}}^{1 / 2}=\widetilde{Z}_{c}^{1 / 2}, \\
Z_{\bar{\omega}}^{1 / 2}=Z_{g}^{-1} Z_{\bar{c}}^{1 / 2} \widetilde{Z}_{c}^{1 / 2}, \quad Z_{\omega}^{1 / 2}=Z_{g} Z_{\bar{c}}^{-1 / 2} \widetilde{Z}_{c}^{1 / 2}, \\
Z_{\bar{M}}=Z_{M}, \quad Z_{g} Z_{\bar{c}}^{-1 / 2} Z_{M}, \quad Z_{\bar{N}}=Z_{g}^{-1} Z_{\bar{c}}^{1 / 2} Z_{M}, \\
Z_{X}=\widetilde{Z}_{c}^{-1 / 2}, \quad Z_{\bar{X}}=Z_{g}^{-2} Z_{\bar{c}} \widetilde{Z}_{c}^{-1 / 2}, \\
Z_{Y}=Z_{\bar{Y}}=Z_{g}^{-1} Z_{\bar{c}}^{1 / 2} \widetilde{Z}_{c}^{-1 / 2} \\
Z_{\theta}=Z_{\bar{\eta}_{i}}=Z_{\eta_{i}}=Z_{g}^{-1} Z_{\bar{c}}^{1 / 2} Z_{c}^{-1 / 2} \\
Z_{\eta}=Z_{\bar{\theta}_{i}}=Z_{g}^{-2} Z_{\bar{c}} Z_{c}^{1 / 2}, \quad Z_{\theta_{i}}=Z_{c}^{-1 / 2} \\
\widetilde{Z}_{L}=Z_{g}^{-1} Z_{\bar{c}}^{1 / 2} \widetilde{Z}_{c}^{-1 / 2}, \quad Z_{L}=Z_{g}^{-1} Z_{\bar{c}}^{1 / 2} Z_{c}^{-1 / 2} \\
Z_{\xi}=Z_{M}, \quad Z_{\Omega}=Z_{\bar{c}}^{1 / 2}
\end{gathered}
$$

with

$$
\begin{aligned}
\widetilde{Z}_{A}^{1 / 2} & =1+\epsilon\left(\frac{a_{0}}{2}+a_{1}\right), \quad Z_{g}=1-\epsilon \frac{a_{0}}{2} \\
\widetilde{Z}_{c}^{1 / 2} & =1-\epsilon \frac{a_{3}+a_{4}}{2}, \quad Z_{\bar{c}}^{1 / 2}=1-\epsilon \frac{a_{3}-a_{4}}{2}, \\
Z_{c}^{1 / 2} & =1+\epsilon\left(\frac{a_{3}-a_{4}}{2}-a_{5}\right) \\
Z_{M} & =1-\epsilon\left(a_{1}+a_{2}+\frac{a_{3}-a_{4}}{2}\right) \\
Z_{\chi} & =1+\epsilon\left(2 a_{1}+2 a_{2}+a_{3}-a_{4}-a_{6}\right) \\
Z_{\alpha} & =1+\epsilon\left(a_{0}+2 a_{4}+a_{7}\right) \\
Z_{\zeta} & =1+\epsilon\left[2 a_{0}-2\left(1+\alpha \frac{\rho}{\zeta}\right) a_{3}+2 a_{4}-2 \alpha \frac{\rho}{\zeta} a_{7}+2 \alpha a_{8}-2 \alpha \frac{\rho}{\zeta} a_{9}+a_{10}\right], \\
Z_{\rho} & =1+\epsilon\left[a_{0}-(1+\alpha) a_{3}+a_{4}-\alpha a_{7}-\left(-\alpha+\frac{\zeta}{\rho}\right)\left(a_{8}-a_{9}\right)+a_{11}\right] \\
Z_{\kappa} & =1-\epsilon\left[2 a_{4}+2 \frac{\rho}{\kappa} a_{8}-2 a_{9}-a_{12}\right] \\
Z_{\beta} & =1-\epsilon\left(a_{0}-2 a_{3}+2 a_{4}+2 a_{5}-a_{13}\right),
\end{aligned}
$$


and

$$
\begin{aligned}
\mathbb{Z}_{\Omega K} & =\mathbb{I}+\epsilon\left(\begin{array}{c|c}
-a_{1}-a_{2}-\frac{1}{2}\left(a_{3}-a_{4}\right) & a_{2} \\
\hline 0 & -a_{1}-\frac{1}{2}\left(a_{3}-a_{4}\right)
\end{array}\right), \\
\mathbb{Z}_{\lambda \tau} & =\mathbb{I}+\epsilon\left(\begin{array}{c|c}
-\frac{1}{2}\left(a_{0}-a_{3}+a_{4}\right)-\alpha a_{8} & a_{8} \\
\hline+\alpha\left(a_{3}+a_{7}+a_{9}\right) & \frac{1}{2}\left(a_{0}-a_{3}+3 a_{4}\right)-a_{9}
\end{array}\right), \\
\mathbb{Z}_{J \sigma} & =\mathbb{I}+\epsilon\left(\begin{array}{c|c}
-a_{0}+a_{3}-a_{4}-\alpha a_{8} & a_{8} \\
\hline+\alpha\left(a_{3}+a_{7}+a_{9}\right) & a_{4}-a_{9}
\end{array}\right) .
\end{aligned}
$$

This concludes the proof of the renormalizability of the complete calssical starting action $\Sigma$.

\section{Conclusion}

In this paper the gluon and ghost propagators have been investigated by taking into account the effects of the Gribov copies as well as of dimension two operators. The output of our results is summarized in Sect.2, where the expressions for the tree level propagators can be found, being in good agreement with the most recent lattice data [31].

Certainly, much work is needed in order to reach a better understanding of the maximal Abelian gauge. Nevertheless, the results which we have obtained enable us to strengthen the fact that the agreement with the lattice data has been obtained only when the effects of the Gribov copies and of the dimension two operators have been simultaneously encoded in the starting Lagrangian, which enjoys the important property of being renormalizable. This point can be better clarified by the following considerations:

\section{- The quantization procedure and the issue of the Gribov copies}

The starting point to analyze Yang-Mills theories at the quantum level is by means of the Faddeev-Popov quantization formula, based on the introduction of a gauge fixing and of the corresponding ghost term. It is known that such a procedure is plagued by the existence of the Gribov copies. A full resolution of this issue, amounting to restrict the domain of integration in the Feynman path integral to the fundamental modular region, is still unavailable. A partial solution to this problem consists of restricting the domain of integration to the Gribov region $\Omega$, which is still affected by Gribov copies. Although this procedure does not eliminate all copies, it has the advantage of being effectively implementable. As we learn from the work of Zwanziger $[22,23]$ in the Landau gauge, the restriction to the region $\Omega$ is achieved through the introduction in the Yang-Mills action of a nonlocal operator, known as the horizon function. This nonlocal operator can be cast in local form by introducing a set of additional localizing fields. Remarkably, the resulting local action turns out to be renormalizable [22, 23]. This procedure has been successfully adapted to the maximal Abelian gauge $[10,13]$. A second point to be noticed is that the introduction of the horizon function in its local form is equivalent to the introduction of a specific dimension two operator. In fact, the Gribov-Zwanziger gap equation $[21,22,23]$ determining the Gribov parameter $\gamma$, namely

$$
\frac{\delta \Gamma}{\delta \gamma}=0
$$

with $\Gamma$ being the 1PI effective action, is equivalent to require the existence of a nonvanishing dimension two condensate. In the case of the Landau gauge, this condensate is given by [22, 23]

$$
\left\langle f^{A B C} A_{\mu}^{A}(x)\left(\phi_{\mu}^{B C}(x)-\bar{\phi}_{\mu}^{B C}(x)\right)\right\rangle \neq 0,
$$

where $\phi_{\mu}^{B C}, \bar{\phi}_{\mu}^{B C}$ stand for the localizing fields and the indices $A, B, C$ belong to the adjoint representation of $S U(2)$. In the case of the maximal Abelian gauge the corresponding condensate is given by $\left\langle\varepsilon^{a b} A_{\mu}(x)\left(\phi_{\mu}^{a b}(x)-\right.\right.$ $\left.\bar{\phi}_{\mu}^{a b}(x)\right\rangle$. The same feature holds in the Coulomb gauge, see [39] for a review.

\section{- Introduction of the dimension two operators}

As mentioned before, the inclusion of the horizon function is equivalent to the introduction of a dimension 
two field operator in the localizing fields. Therefore, we can look for other dimension two operators which can be added to the theory, provided one is able to maintain renormalizability. From this point of view, the introduction of the three dimension two operators $\mathcal{O}_{A^{2}}=\frac{1}{2} A_{\mu}^{a} A_{\mu}^{a}, \mathcal{O}_{\bar{f} f}=\left(\bar{\phi}_{i}^{b} \phi_{i}^{b}-\bar{\omega}_{i}^{b} \omega_{i}^{b}-\bar{c}^{b} c^{b}\right), \mathcal{O}_{\text {ghost }}=\left(\varepsilon^{a b} \bar{c}^{a} c^{b}\right)$ looks very natural. It is remarkable that these three operators can be simultaneously added to the horizon term in a way which preserves renormalizability. We also notice that all three operators considered here have their analogue in the Landau gauge, see [7, 28] and refs. therein. In much the same way as the horizon function, these operators carry nonperturbative information, encoded in the corresponding condensates.

The good agreement of our results with the lattice data can be taken as evidence of the fact these dimension two operators play a relevant role in the infrared. For example, without the introduction of the two operators $\mathcal{O}_{\bar{f} f}, \mathcal{O}_{\text {ghost }}$, the infrared behavior of the off-diagonal ghost propagator would be deeply different from that of eq.(12). Instead, it would have displayed an enhanced behavior of the type $1 / k^{4}$, as reported in our previous investigation [10], where only the horizon function and the gluon condensate $\mathcal{O}_{A^{2}}$ were taken into account. The same occurs for the diagonal gluon propagator, eq.(8). Without the introduction of $\mathcal{O}_{\bar{f} f}$ it would be vanishing at $k=0$.

We remark that the same features have been detected in the Landau gauge, where the most recent lattice data $[40,41,42]$ point towards a finite and nonvanishing gluon propagator at $k=0$, while exhibiting a less enhanced ghost propagator. As discussed in [28] these features can be accounted for by considering the effects of dimension two operators, which nicely fit within the Gribov-Zwanziger framework.

\section{Acknowledgments}

S. P. Sorella thanks D. Dudal, A. Cucchieri, T. Mendes, N. Vandersickel and D. Zwanziger for useful discussion. The Conselho Nacional de Desenvolvimento Científico e Tecnológico (CNPq-Brazil), the Faperj, Fundação de Amparo à Pesquisa do Estado do Rio de Janeiro, the SR2-UERJ and the Coordenação de Aperfeiçoamento de Pessoal de Nível Superior (CAPES) are gratefully acknowledged for financial support.

\section{References.}

[1] G. 't Hooft, Nucl. Phys. B 190 (1981) 455.

[2] A. S. Kronfeld, G. Schierholz and U. J. Wiese, Nucl. Phys. B 293 (1987) 461.

[3] A. S. Kronfeld, M. L. Laursen, G. Schierholz and U. J. Wiese, Phys. Lett. B 198 (1987) 516.

[4] A. R. Fazio, V. E. R. Lemes, M. S. Sarandy and S. P. Sorella, Phys. Rev. D 64, 085003 (2001) [arXiv:hepth/0105060].

[5] V. E. R. Lemes, M. S. Sarandy and S. P. Sorella, J. Phys. A 36, 7211 (2003) [arXiv:hep-th/0206251].

[6] D. Dudal, V. E. R. Lemes, M. S. Sarandy, S. P. Sorella and M. Picariello, JHEP 0212, 008 (2002) [arXiv:hepth/0211007].

[7] D. Dudal et al., Phys. Lett. B 569, 57 (2003) [arXiv:hep-th/0306116].

[8] D. Dudal, J. A. Gracey, V. E. R. Lemes, M. S. Sarandy, R. F. Sobreiro, S. P. Sorella and H. Verschelde, Phys. Rev. D 70, 114038 (2004) [arXiv:hep-th/0406132].

[9] D. Dudal, J. A. Gracey, V. E. R. Lemes, M. S. Sarandy, R. F. Sobreiro, S. P. Sorella and H. Verschelde, Braz. J. Phys. 37, 406 (2007) [arXiv:hep-th/0501227].

[10] M. A. L. Capri, V. E. R. Lemes, R. F. Sobreiro, S. P. Sorella and R. Thibes, Phys. Rev. D 72, 085021 (2005) [arXiv:hep-th/0507052].

[11] J. A. Gracey, JHEP 0504, 012 (2005) [arXiv:hep-th/0504051]. 
[12] M. A. L. Capri et al., Braz. J. Phys. 37, 591 (2007) [arXiv:hep-th/0603167].

[13] M. A. L. Capri, V. E. R. Lemes, R. F. Sobreiro, S. P. Sorella and R. Thibes, Phys. Rev. D 74, 105007 (2006) [arXiv:hep-th/0609212].

[14] M. A. L. Capri, D. Dudal, J. A. Gracey, S. P. Sorella and H. Verschelde, arXiv:0708.4303 [hep-th].

[15] Y. Nambu, Phys. Rev. D10 (1974) 4262;

G. 't Hooft, High Energy Physics EPS Int. Conference, Palermo 1975, ed. A. Zichichi;

S. Mandelstam, Phys. Rept. 23 (1976) 245.

[16] Z. F. Ezawa and A. Iwazaki, Phys. Rev. D 25 (1982) 2681.

[17] T. Suzuki and I. Yotsuyanagi, Phys. Rev. D 42 (1990) 4257.

[18] T. Suzuki, S. Hioki, S. Kitahara, S. Kiura, Y. Matsubara, O. Miyamura and S. Ohno, Nucl. Phys. Proc. Suppl. 26 (1992) 441.

[19] S. Hioki, S. Kitahara, S. Kiura, Y. Matsubara, O. Miyamura, S. Ohno and T. Suzuki, Phys. Lett. B 272 (1991) 326 [Erratum-ibid. B 281 (1992) 416].

[20] F. Bruckmann, T. Heinzl, A. Wipf and T. Tok, . Nucl. Phys. B 584, 589 (2000) [arXiv:hep-th/0001175].

[21] V. N. Gribov, Nucl. Phys. B 139 (1978) 1.

[22] D. Zwanziger, Nucl. Phys. B 323, 513 (1989).

[23] D. Zwanziger, Nucl. Phys. B 399, 477 (1993).

[24] K. I. Kondo, T. Murakami, T. Shinohara and T. Imai, Phys. Rev. D 65, 085034 (2002) [arXiv:hep-th/0111256].

[25] M. Schaden, arXiv:hep-th/9909011.

[26] K. I. Kondo, Phys. Lett. B 514, 335 (2001) [arXiv:hep-th/0105299].

[27] D. Dudal and H. Verschelde, J. Phys. A 36, 8507 (2003) [arXiv:hep-th/0209025].

[28] D. Dudal, S. P. Sorella, N. Vandersickel and H. Verschelde, arXiv:0711.4496 [hep-th].

[29] K. Amemiya and H. Suganuma, Phys. Rev. D 60 (1999) 114509.

[30] V. G. Bornyakov, M. N. Chernodub, F. V. Gubarev, S. M. Morozov and M. I. Polikarpov, Phys. Lett. B 559 (2003) 214.

[31] T. Mendes, A. Cucchieri and A. Mihara, AIP Conf. Proc. 892, 203 (2007) [arXiv:hep-lat/0611002].

[32] M. A. L. Capri et. al., in preparation.

[33] J. M. Cornwall, Phys. Rev. D 26, 1453 (1982).

[34] J. M. Cornwall and W. S. Hou, Phys. Rev. D 34, 585 (1986).

[35] M. Lavelle, Phys. Rev. D 44 (1991) 26.

[36] A. C. Aguilar and J. Papavassiliou, Eur. Phys. J. A 35, 189 (2008) [arXiv:0708.4320 [hep-ph]].

[37] N. Maggiore and M. Schaden, Phys. Rev. D 50, 6616 (1994) [arXiv:hep-th/9310111].

[38] O. Piguet and S. P. Sorella, "Algebraic renormalization: Perturbative renormalization, symmetries and anomalies," Lect. Notes Phys. M28, 1 (1995).

[39] D. Zwanziger, Braz. J. Phys. 37, 127 (2007).

[40] A. Cucchieri and T. Mendes, arXiv:0710.0412 [hep-lat].

[41] I. L. Bogolubsky, E. M. Ilgenfritz, M. Muller-Preussker and A. Sternbeck, arXiv:0710.1968 [hep-lat].

[42] A. Cucchieri and T. Mendes, arXiv:0712.3517 [hep-lat]. 